\title{
Lawvere completeness in Topology
}

\author{
Maria Manuel Clementino and Dirk Hofmann*
}

October 31, 2018

\begin{abstract}
It is known since 1973 that Lawvere's notion of (Cauchy-)complete enriched category is meaningful for metric spaces: it captures exactly Cauchy-complete metric spaces. In this paper we introduce the corresponding notion of Lawvere completeness for $(\mathbb{T}, \mathrm{V})$-categories and show that it has an interesting meaning for topological spaces and quasi-uniform spaces: for the former ones means weak sobriety while for the latter means Cauchy completeness. Further, we show that $\mathrm{V}$ has a canonical $(\mathbb{T}, \mathrm{V})$-category structure which plays a key role: it is Lawvere-complete under reasonable conditions on the setting; permits us to define a Yoneda embedding in the realm of $(\mathbb{T}, \mathrm{V})$-categories.

Mathematics Subject Classification (2000): 18A05, 18D15, 18D20, 18B35, 18C15, 54E15, $54 \mathrm{E} 50$.
\end{abstract}

Key words: $\mathrm{V}$-category, bimodule, monad, $(\mathbb{T}, \mathrm{V})$-category, completeness.

\section{Introduction}

Lawvere in his 1973 paper Metric spaces, generalized logic, and closed categories formulates a notion of complete $\mathrm{V}$-category and shows that for (generalised) metric spaces it means Cauchy completeness. This notion of completeness deserved the attention of the categorical community, and the notion of Cauchy-complete category, or Freyd-Karoubi complete category is well-known, mostly in the context of Ab-enriched categories. However, it never got the attention of the topological community. In this paper we interpret Lawvere's completeness in topological settings. We extend Lawvere's notion of complete $\mathrm{V}$-category to the (topological) setting of $(\mathbb{T}, \mathrm{V})$-categories (for a symmetric and unital quantale $\mathrm{V}$ ), and show that it encompasses well-known notions in topological categories, meaning weakly sober space in the category of topological spaces and continuous maps, weakly sober approach space in the category of approach spaces and non-expansive maps, and Cauchy-completeness in the category of quasi-uniform spaces and uniformly continuous maps.

We present also a first step towards a possible construction of completion. Indeed, in the setting of $\mathrm{V}$-categories, it is well-known that the completion of a $\mathrm{V}$-category may be built out of the Yoneda embedding $X \rightarrow \mathrm{V}^{X^{\text {op }}}$. In the $(\mathbb{T}, \mathrm{V})$-setting, we could prove that $\mathrm{V}$ has a canonical $(\mathbb{T}, \bigvee)$-categorical structure and that every $(\mathbb{T}, \bigvee)$-category $X$ has a canonical dual

${ }^{*}$ The authors acknowledge partial financial assistance by Centro de Matemática da Universidade de Coimbra/FCT and Unidade de Investigação e Desenvolvimento Matemática e Aplicações da Universidade de Aveiro/FCT. 
$X^{\mathrm{op}}$. Using this structure and the free Eilenberg-Moore algebra structure $|X|$ on $T X$, we get two "Yoneda-like" morphisms

$$
X \rightarrow \mathrm{V}^{X^{\mathrm{op}}} \text { and } X \rightarrow \mathrm{V}^{|X|} .
$$

For the latter one we prove a Yoneda Lemma (see 4.2).

Furthermore, we show that, under suitable conditions, $\mathrm{V}$ is a Lawvere-complete $(\mathbb{T}, \mathrm{V})$ category, a first step towards a completion construction which will be the subject of a forthcoming paper.

In order to make the presentation of this paper smoother, in Section 1 we recall the notions and properties of $\mathrm{V}$-categories we will generalize throughout. First we introduce $\mathrm{V}$-categories and V-bimodules, and define Lawvere-complete $\mathrm{V}$-categories, for a commutative and unital quantale $\mathrm{V}$. $\mathrm{V}$ is then naturally equipped with the $\mathrm{V}$-categorical structure hom. We give a direct proof of Lawvere completeness of the $\mathrm{V}$-category ( $V$, hom).

In Section 2 we describe our basic setting for the study of $(\mathbb{T}, V)$-categories and introduce them. We describe Kleisli composition in the category V-Mat of V-valued matrices and define $(\mathbb{T}, \mathrm{V})$-bimodule. Although $(\mathbb{T}, \mathrm{V})$-bimodules do not compose in general, one can still formulate and study the notion of Lawvere-complete $(\mathbb{T}, V)$-category.

Similarly to what was done in $\mathrm{V}$-categories, we define a canonical $(\mathbb{T}, \mathrm{V})$-categorical structure on $\mathrm{V}$, as the composition of hom with the (canonical) $\mathbb{T}$-algebra structure on $\mathrm{V}$ described by Manes in [21]. This is the subject of Section 3. In addition we also prove that, under some conditions, the $(\mathbb{T}, \mathrm{V})$-category $\mathrm{V}$ is Lawvere-complete.

In Section 1 we present the Yoneda embedding for $\mathrm{V}$-categories as a subproduct of the fact that a $\mathrm{V}$-matrix $\psi: X \rightarrow Y$ between $\mathrm{V}$-categories $(X, a)$ and $(Y, b)$ is a $\mathrm{V}$-bimodule if and only if, as a map $\psi: X^{\mathrm{op}} \otimes Y \rightarrow \mathrm{V}$, is a $\mathrm{V}$-functor (Theorem 1.5); then the monoidal-closed structure of $\mathrm{V}$-Cat gives us the Yoneda Functor $X \rightarrow \mathrm{V}^{X^{\text {op }}}$. In the $(\mathbb{T}, \mathrm{V})$-setting this construction becomes more elaborated (see Theorem 3.3): a $\vee$-matrix $\psi: T X \mapsto Y$ is a $(\mathbb{T}, \mathrm{V})$-bimodule $\psi:(X, a) \rightarrow(Y, b)$ if and only if both $\psi:|X| \otimes Y \rightarrow \vee$ and $\psi: X^{\text {op }} \otimes Y \rightarrow \vee$ are $(\mathbb{T}, \mathrm{V})-$ functors. Thus, given a $(\mathbb{T}, \mathrm{V})$-category $(X, a)$, the $(\mathbb{T}, \mathrm{V})$-bimodule $a: X \rightarrow X$ gives rise to two $\operatorname{Yoneda}(\mathbb{T}, \mathrm{V})$-functors $X \rightarrow \mathrm{V}^{X^{\mathrm{op}}}$ and $X \rightarrow \mathrm{V}^{|X|}$.

In Section 5 we present the announced topological examples, with the exception of quasiuniform spaces, which are presented in the Appendix, due to the fact that their presentation as lax algebras does not fit in the ( $\mathbb{T}, \mathrm{V}$ )-setting (as shown in [20]).

Acknowledgments. We are grateful to Francis Borceux for his enlightening proof of completeness of the $\mathrm{V}$-category $\mathrm{V}$. The work on this paper was started while both authors were visiting Walter Tholen in May 2004, at York University, Canada, and benefited from further visits in January 2006 and February 2007. We thank Walter Tholen for fruitful discussions on the subject of this paper.

\section{The category of $V$-categories}

Although the material of this section can be found essentially on [17, we find that its inclusion here may enlighten the corresponding - but more technical - notions and results for $(\mathbb{T}, \bigvee)$ - 
categories presented in the forthcoming sections.

1.1 V. Throughout $\mathrm{V}$ is a (commutative and unital) quantale. In other words, $\mathrm{V}$ is a complete lattice equipped with a symmetric and associative tensor product $\otimes$, with unit $k$, and with right adjoint hom; that is, for each $u, v, w \in \mathrm{V}$,

$$
u \otimes v \leq w \Longleftrightarrow v \leq \operatorname{hom}(u, w) .
$$

Considered as a (thin) category, $\mathrm{V}$ is said to be symmetric monoidal-closed. If $k$ is the bottom element $\perp$ of $\mathrm{V}$, then $\mathrm{V}=1$ is the trivial lattice. Throughout this paper we assume that $\mathrm{V}$ is non-trivial, i.e. $k \neq \perp$.

Every non-trivial Heyting algebra - with $\otimes=\wedge$ and $k=\top$ the top element - is an example of such a lattice, in particular the two-element chain $2=\{$ false $\models$ true $\}$, with the monoidal structure given by "\&" (and) and "true". The complete real half-line $P=[0, \infty]$, with the categorical structure induced by the relation $\geq$ (i.e., $a \rightarrow b$ means $a \geq b$ ), admits several interesting monoidal structures. First of all, with $\wedge=\max$ it is a Heyting algebra $P_{\max }$. Another possible choice for $\otimes$ is + ; we denote $P$ equipped with this tensor by $P_{+}$. Note that in this example the right adjoint hom is given by truncated minus: $\operatorname{hom}(u, v)=\max \{v-u, 0\}$.

1.2 V-Mat. The category $\mathrm{V}$-Mat of $\mathrm{V}$-matrices [3, 10] has sets as objects, and a morphism $r: X \rightarrow Y$ in $\mathrm{V}$-Mat is a map $r: X \times Y \rightarrow \mathrm{V}$. Composition of $\mathrm{V}$-matrices $r: X \rightarrow Y$ and $s: Y \rightarrow Z$ is defined as matrix multiplication:

$$
s \cdot r(x, z)=\bigvee_{y \in Y} r(x, y) \otimes s(y, z) .
$$

The identity arrow $1_{X}: X \mapsto X$ in $\mathrm{V}$-Mat is the $\mathrm{V}$-matrix which sends all diagonal elements $(x, x)$ to $k$ and all other elements to the bottom element $\perp$ of $\mathrm{V}$. In fact, each Set-map $f: X \rightarrow Y$ can be interpreted as the $\mathrm{V}$-matrix

$$
f: X \mapsto Y, f(x, y)= \begin{cases}k & \text { if } f(x)=y \\ \perp & \text { else. }\end{cases}
$$

To keep notation simple, in the sequel we will write $f: X \rightarrow Y$ rather then $f: X \rightarrow Y$ for a $\mathrm{V}$-matrix induced by a map. The formula for matrix composition becomes considerably easier if one of the $\mathrm{V}$-matrices is a Set-map:

$$
s \cdot f(x, z)=s(f(x), z), \quad g \cdot r(x, z)=\bigvee_{y \in g^{-1}(z)} r(x, y)
$$

for maps $f: X \rightarrow Y$ and $g: Y \rightarrow Z$ and $\mathrm{V}$-matrices $r: X \mapsto Y$ and $s: Y \rightarrow Z$.

The complete order on $\mathrm{V}$ induces a complete order on $\mathrm{V}$-Mat $(X, Y)=\mathrm{V}^{X \times Y}$ : for $\mathrm{V}$-matrices $r, r^{\prime}: X \rightarrow Y$ we define

$$
r \leq r^{\prime}: \Longleftrightarrow \forall x \in X \forall y \in Y r(x, y) \leq r^{\prime}(x, y) .
$$

The transpose $r^{\circ}: Y \rightarrow X$ of a $\mathrm{V}$-matrix $r: X \rightarrow Y$ is defined by $r^{\circ}(y, x)=r(x, y)$. It is easy to see that ()$^{\circ}: V-\operatorname{Mat}(X, Y) \rightarrow V$-Mat $(Y, X)$ is order-preserving, and

$$
1_{X}^{\circ}=1_{X}, \quad(s \cdot r)^{\circ}=r^{\circ} \cdot s^{\circ}, \quad r^{\circ 0}=r .
$$


For each Set-map $f: X \rightarrow Y$ we have $1_{X} \leq f^{\circ} \cdot f$ and $f \cdot f^{\circ} \leq 1_{Y}$, i.e. $f$ is left adjoint to $f^{\circ}$ and we write $f \dashv f^{\circ}$. In general, given $\vee$-matrices $r: X \mapsto Y$ and $s: Y \mapsto X$, we say that $r$ is left adjoint to $s$ (and that $s$ is right adjoint to $r$ ) if $1_{X} \leq s \cdot r$ and $1_{Y} \geq r \cdot s$.

Lemma. Let $\mathrm{V}$ be a quantale and $r, r^{\prime}: X \mapsto Y$ and $s, s^{\prime}: Y \mapsto X$ be $\mathrm{V}$-matrices such that $r \dashv s$ and $r^{\prime} \dashv s^{\prime}$. Then $r \leq r^{\prime}$ if and only if $s^{\prime} \leq s$. Consequently, if $r \leq r^{\prime}$ and $s \leq s^{\prime}$, then $r=r^{\prime}$ and $s=s^{\prime}$.

As another consequence of the lemma above we have that left and right adjoints are uniquely determined by $s$ (respectively $r$ ). Therefore we say that $r$ is left adjoint if it has a right adjoint $s$, and likewise, $s$ is right adjoint if it has a left adjoint $r$. In pointwise notation, we have $r \dashv s$ if and only if

$$
\begin{aligned}
& \forall x \in X \bigvee_{y \in Y} r(x, y) \otimes s(y, x) \geq k \\
& \forall x \in X \forall y, y^{\prime} \in Y s(y, x) \otimes r\left(x, y^{\prime}\right) \leq \begin{cases}\perp & \text { if } y \neq y^{\prime} \\
k & \text { if } y=y^{\prime}\end{cases}
\end{aligned}
$$

which, by symmetry of $\otimes$, is equivalent to

$$
\begin{aligned}
& \forall x \in X \bigvee_{y \in Y} r(x, y) \otimes s(y, x)=k \\
& \forall x \in X \forall y, y^{\prime} \in Y\left(y \neq y^{\prime} \Rightarrow s(y, x) \otimes r\left(x, y^{\prime}\right)=\perp\right)
\end{aligned}
$$

Our next example shows that there exist indeed left adjoint $\mathrm{V}$-matrices which are not induced by Set-maps.

Example. Consider a set $X$ and the Boolean algebra $\mathrm{V}=P X$ the powerset of $X$. Define a V-matrix $r: 1 \longrightarrow X$ by putting $r(\star, x)=\{x\}$ for $x \in X$. Then

$$
r^{\circ} \cdot r(\star, \star)=\bigcup_{x \in X}\{x\}=X \quad \text { and } \quad r \cdot r^{\circ}(x, y)=\{x\} \cap\{y\}= \begin{cases}\emptyset & \text { if } x \neq y \\ \{x\} & \text { if } x=y\end{cases}
$$

hence $r \dashv r^{\circ}$. But $r$ is not a Set-map unless $X$ has at most one element.

We wish to characterise those quantales $\mathrm{V}$ where the class of left adjoint $\mathrm{V}$-matrices coincides with the class of Set-maps. In order to do so we introduce some notation. Let $u, v \in \mathrm{V}$. We say that $v$ is a $\otimes$-complement of $u$ if

$$
u \vee v=k \quad \text { and } \quad u \otimes v=\perp .
$$

Clearly, each $u \in \mathrm{V}$ has at most one $\otimes$-complement. Moreover, if $u$ is $\otimes$-complemented (i.e. has a $\otimes$-complement $v$ ), then

$$
u=u \otimes k=u \otimes(u \vee v)=(u \otimes u) \vee(u \otimes v)=u \otimes u,
$$

that is, $u$ is idempotent. Our next result generalises [14, 2.14]. 
Proposition. Let $\mathrm{V}$ be a quantale. Each left adjoint $\mathrm{V}$-matrix is a Set-map if and only if $k$ and $\perp$ are the only $\otimes$-complemented elements of $\mathrm{V}$ and

$$
\forall u, v \in \mathrm{V}(u \otimes v=k \Rightarrow u=k=v)
$$

Proof. Assume first that each left adjoint V-matrix is a Set-map. Let $u, v \in \mathrm{V}$. If $u \otimes v=k$, then $u \dashv v$, and we have $u=v=k$. Suppose that $u \vee v=k$ and $u \otimes v=\perp$. Let $X=\{u, v\}$ and define $r: 1 \longmapsto X$ with $r(\star, u)=u$ and $r(\star, v)=v$. Then $r \dashv r^{\circ}$ and, by assumption, $u=k$ or $v=k$.

Let $r: X \mapsto Y$ and $s: Y \mapsto X$ be $\mathrm{V}$-matrices such that $r \dashv s$. Let $x \in X$. There is some $y \in Y$ such that $r(x, y) \otimes s(y, x)>\perp 1$. Then

$$
k=(r(x, y) \otimes s(y, x)) \vee \bigvee_{y^{\prime} \neq y}\left(r\left(x, y^{\prime}\right) \otimes s\left(y^{\prime}, x\right)\right)
$$

and

$$
r(x, y) \otimes s(y, x) \otimes \bigvee_{y^{\prime} \neq y} r\left(x, y^{\prime}\right) \otimes s\left(y^{\prime}, x\right)=\bigvee_{y^{\prime} \neq y} r(x, y) \otimes s(y, x) \otimes r\left(x, y^{\prime}\right) \otimes s\left(y^{\prime}, x\right)=\perp .
$$

Hence, by assumption, $r(x, y)=k=s(y, x)$ and $r\left(x, y^{\prime}\right) \otimes s\left(y^{\prime}, x\right)=\perp$ for all $y^{\prime} \neq y$. We have shown that, for each $x \in X$, there exists exactly one $y \in Y$ with $r(x, y)=k=s(y, x)$. Consider now $f: X \rightarrow Y$ which assigns to $x$ this unique $y$. Clearly, $f \leq r$, but also $f^{\circ} \leq s$ since

$$
f^{\circ}(y, x)=k \Rightarrow f(x)=y \quad \Rightarrow \quad s(y, x)=k .
$$

The assertion follows now from the previous lemma.

1.3 V-categories. V-enriched categories were introduced and studied in [11, 17] in the more general context of symmetric monoidal-closed categories. For a very nice presentation of this material we refer to [18]. In the next subsections we recall some well-known facts about $\mathrm{V}$ categories, which will serve as a guideline for our study of $(\mathbb{T}, \mathrm{V})$-categories.

A $\mathrm{V}$-enriched category (or simply $\mathrm{V}$-category) is a pair $(X, a)$ with $X$ a set and $a: X \mapsto X$ a V-matrix such that

$$
1_{X} \leq a \cdot a \text { and } a \cdot a \leq a
$$

equivalently, the map $a: X \times X \rightarrow \mathrm{V}$ satisfies the following conditions:

(R) for each $x \in X, k \leq a(x, x)$;

(T) for each $x, x^{\prime}, x^{\prime \prime} \in X, a\left(x, x^{\prime}\right) \otimes a\left(x^{\prime}, x^{\prime \prime}\right) \leq a\left(x, x^{\prime \prime}\right)$.

Given $\mathrm{V}$-categories $(X, a)$ and $(Y, b)$, a $\vee$-functor $f:(X, a) \rightarrow(Y, b)$ is a map $f: X \rightarrow Y$ such that, for each $x, x^{\prime} \in X, a\left(x, x^{\prime}\right) \leq b\left(f(x), f\left(x^{\prime}\right)\right)$. $\mathrm{V}$-categories and $\mathrm{V}$-functors are the objects and morphisms of the category $\mathrm{V}$-Cat. Finally, given a $\mathrm{V}$-category $X=(X, a)$, the dual category $X^{\text {op }}$ of $X$ is defined by $X^{\text {op }}=\left(X, a^{\circ}\right)$.

We remark that $\mathrm{V}$-Cat is actually a closed category since the tensor product on $\mathrm{V}$ can be naturally transported to $\mathrm{V}$-Cat. More precisely, for $\mathrm{V}$-categories $X=(X, a)$ and $Y=(Y, b)$, we

\footnotetext{
${ }^{1}$ Since $\perp<k$. The assertion of the proposition is trivially true if $k=\perp$
} 
put $X \otimes Y=(X \times Y, a \otimes b)$ where $a \otimes b\left((x, y),\left(x^{\prime}, y^{\prime}\right)\right)=a\left(x, x^{\prime}\right) \otimes b\left(y, y^{\prime}\right)$ for all $x, x^{\prime} \in X$ and $y, y^{\prime} \in Y$. Then, for each $\mathrm{V}$-category $X=(X, a)$, the functor $X \otimes_{-}: \mathrm{V}$-Cat $\rightarrow \mathrm{V}$-Cat has a right adjoint ${ }_{-}^{X}$ defined by $Y^{X}=\left(\mathrm{V}_{-} \mathrm{Cat}(X, Y), d\right)$ with $d(f, g)=\bigwedge_{x \in X} a(f(x), g(x))$.

Being monoidal-closed, $\mathrm{V}$ has a natural structure as $\mathrm{V}$-category:

$$
\text { hom }: \mathrm{V} \rightarrow \mathrm{V} \text {. }
$$

Indeed, for $u, v, w \in \mathrm{V}$,

$$
k \otimes v=v \Rightarrow k \leq \operatorname{hom}(v, v),
$$

$u \otimes(\operatorname{hom}(u, v) \otimes \operatorname{hom}(v, w)) \leq v \otimes \operatorname{hom}(v, w) \leq w \Rightarrow \operatorname{hom}(u, v) \otimes \operatorname{hom}(v, w) \leq \operatorname{hom}(u, w)$,

that is, $1_{\mathrm{V}} \leq \mathrm{hom}$ and hom $\cdot$ hom $\leq$ hom.

For $\mathrm{V}=2$, with the usual notation $x \leq x^{\prime}: \Longleftrightarrow a\left(x, x^{\prime}\right)=$ true, axioms $(\mathrm{R})$ and $(\mathrm{T})$ read as

$$
\forall x \in X \text { true } \models x \leq x \quad \text { and } \quad \forall x, x^{\prime}, x^{\prime \prime} \in X x \leq x^{\prime} \& x^{\prime} \leq x^{\prime \prime} \models x \leq x^{\prime \prime},
$$

that is, $(X, \leq)$ is an ordered set2. A 2-functor is a map $f:(X, \leq) \rightarrow(Y, \leq)$ between ordered sets such that

$$
\forall x, x^{\prime} \in X x \leq x^{\prime} \models f(x) \leq f\left(x^{\prime}\right) ;
$$

that is, $f$ is a monotone map. Hence 2-Cat is equivalent to the category Ord of ordered sets and monotone maps.

A $\mathrm{P}_{+}$-category is a set $X$ endowed with a map $a: X \times X \rightarrow \mathrm{P}_{+}$such that

$$
\forall x \in X 0 \geq a(x, x) \quad \text { and } \quad \forall x, x^{\prime}, x^{\prime \prime} \in X a\left(x, x^{\prime}\right)+a\left(x^{\prime}, x^{\prime \prime}\right) \geq a\left(x, x^{\prime \prime}\right) ;
$$

that is, $a: X \times X \rightarrow \mathrm{P}_{+}$is a (generalised) metric on $X$. A $\mathrm{P}_{+}$-functor is a map $f:(X, a) \rightarrow(Y, b)$ between metric spaces satisfying the following inequality:

$$
\forall x, x^{\prime} \in X a\left(x, x^{\prime}\right) \geq b\left(f(x), f\left(x^{\prime}\right)\right),
$$

which means precisely that $f$ is a non-expansive map. Therefore the category $\mathrm{P}_{+}$-Cat coincides with the category Met of metric spaces and non-expansive maps. (For more details, see [18, 10].)

For $\mathrm{V}=\mathrm{P}_{\max }$, the transitivity axiom $(\mathrm{T})$ reads as

$$
\max \left\{a\left(x, x^{\prime}\right), a\left(x^{\prime}, x^{\prime \prime}\right)\right\} \geq a\left(x, x^{\prime \prime}\right),
$$

hence the category $\mathrm{P}_{\max }$-Cat coincides with the category UMet of (generalised) ultrametric spaces and non-expansive maps.

1.4 V-bimodules. Given V-categories $(X, a)$ and $(Y, b)$, a bimoduld $\psi:(X, a) \rightarrow \rightarrow(Y, b)$ is a V-matrix $\psi: X \mapsto Y$ such that $\psi \cdot a \leq \psi$ and $b \cdot \psi \leq \psi$; that is, for each $x, x^{\prime} \in X$ and $y, y^{\prime} \in Y$,

$$
a\left(x, x^{\prime}\right) \otimes \psi\left(x^{\prime}, y\right) \leq \psi(x, y) \quad \text { and } \quad \psi\left(x, y^{\prime}\right) \otimes b\left(y^{\prime}, y\right) \leq \psi(x, y) .
$$

It is easy to verify that bimodules compose and that V-categorical structures are themselves bimodules. In fact, they are the identities for the composition of bimodules, that is, for any bimodule $\psi:(X, a) \multimap(Y, b), \psi \cdot a=\psi$ and $b \cdot \psi=\psi$. Therefore, $\mathrm{V}$-categories and $\mathrm{V}$-bimodules constitute a category, which we will denote by V-Mod. The category V-Mod inherits the bicategorical structure of V-Mat via the forgetful functor $\mathrm{V}$-Mod $\rightarrow$ V-Mat.

\footnotetext{
${ }^{2}$ Note that we do not require $\leq$ to be anti-symmetric.

${ }^{3}$ Also known as profunctor or distributor (see [2, 5, 26]).
} 
1.5 V-functors as $\mathrm{V}$-bimodules. Any $\mathrm{V}$-functor $f:(X, a) \rightarrow(Y, b)$ defines a pair of matrices $f_{*}:(X, a) \longrightarrow(Y, b)$ and $f^{*}:(Y, b) \longrightarrow(X, a)$, with $f_{*}=b \cdot f$ and $f^{*}=f^{\circ} \cdot b$, that is $f_{*}(x, y)=b(f(x), y)$ and $f^{*}(y, x)=b(y, f(x))$, which are in fact bimodules: for every $x, x^{\prime} \in X$ and $y, y^{\prime} \in Y$,

$$
\begin{aligned}
a\left(x, x^{\prime}\right) \otimes f_{*}\left(x^{\prime}, y\right) & =a\left(x, x^{\prime}\right) \otimes b\left(f\left(x^{\prime}\right), y\right) \leq b\left(f(x), f\left(x^{\prime}\right)\right) \otimes b\left(f\left(x^{\prime}\right), y\right) \leq b(f(x), y), \\
f_{*}\left(x, y^{\prime}\right) \otimes b\left(y^{\prime} y\right) & =b\left(f(x), y^{\prime}\right) \otimes b\left(y^{\prime}, y\right) \leq b(f(x), y)
\end{aligned}
$$

and similarly for $f^{*}$.

Moreover, the bimodules $f_{*}$ and $f^{*}$ form an adjunction, as we show next. We recall first that, given bimodules $\varphi:(X, a) \multimap(Y, b)$ and $\psi:(Y, b) \rightarrow(X, a), \varphi$ is left adjoint to $\psi, \varphi \dashv \psi$, if $1_{(X, a)} \leq \psi \cdot \varphi$ and $\varphi \cdot \psi \leq 1_{(Y, b)}$, i.e. $a \leq \psi \cdot \varphi$ and $\varphi \cdot \psi \leq b$. It is now straightforward to check that $f_{*} \dashv f^{*}$, since, for $x, x^{\prime} \in X$ and $y, y^{\prime} \in Y$, the inequality

$$
a\left(x, x^{\prime}\right) \leq \bigvee_{y \in Y} f_{*}(x, y) \otimes f^{*}\left(y, x^{\prime}\right)=\bigvee_{y \in Y} b(f(x), y) \otimes b\left(y, f\left(x^{\prime}\right)\right)=b\left(f(x), f\left(x^{\prime}\right)\right)
$$

follows from $\mathrm{V}$-functoriality of $f$, while

$$
\bigvee_{x \in X} f^{*}(y, x) \otimes f_{*}\left(x, y^{\prime}\right)=\bigvee_{x \in X} b(y, f(x)) \otimes b\left(f(x), y^{\prime}\right) \leq b\left(y, y^{\prime}\right)
$$

follows from the associativity axiom for $\mathrm{V}$-categories. A quite different connection between functors and bimodules offers the following

Theorem. For $\mathrm{V}$-categories $X=(X, a)$ and $Y=(Y, b)$ and $a \mathrm{~V}$-matrix $\psi: X \rightarrow Y$, the following conditions are equivalent:

(i) $\psi: X \rightarrow Y$ is a bimodule;

(ii) $\psi: X^{\mathrm{op}} \otimes Y \rightarrow \mathrm{V}$ is a $\mathrm{V}$-functor.

Proof. (i) $\Rightarrow$ (ii): For $x, x^{\prime} \in X$ and $y, y^{\prime} \in Y$,

$$
\begin{aligned}
\psi(x, y) \otimes a^{\circ}\left(x, x^{\prime}\right) \otimes b\left(y, y^{\prime}\right) & =a\left(x^{\prime}, x\right) \otimes \psi(x, y) \otimes b\left(y, y^{\prime}\right) \\
& \leq \psi\left(x^{\prime}, y\right) \otimes b\left(y, y^{\prime}\right) \\
& \leq \psi\left(x^{\prime}, y^{\prime}\right),
\end{aligned}
$$

hence

$$
a^{\circ}\left(x, x^{\prime}\right) \otimes b\left(y, y^{\prime}\right) \leq \operatorname{hom}\left(\psi(x, y), \psi\left(x^{\prime}, y^{\prime}\right)\right) .
$$

(ii) $\Rightarrow$ (i): For $x, x^{\prime} \in X$ and $y, y^{\prime} \in Y$,

$$
\begin{aligned}
a\left(x, x^{\prime}\right) \otimes \psi\left(x^{\prime}, y\right) & \leq \psi\left(x^{\prime}, y\right) \otimes a^{\circ}\left(x^{\prime}, x\right) \otimes b(y, y) \\
& \leq \psi(x, y)
\end{aligned}
$$

that is $a \cdot \psi \leq \psi$, and

$$
\begin{aligned}
\psi\left(x, y^{\prime}\right) \otimes b\left(y^{\prime}, y\right) & \leq \psi\left(x, y^{\prime}\right) \otimes a^{\circ}(x, x) \otimes b\left(y^{\prime}, y\right) \\
& \leq \psi(x, y)
\end{aligned}
$$

that is $\psi \cdot b \leq \psi$. 
Corollary. There is a $\mathrm{\vee}$-functor $\ulcorner a\urcorner: X \rightarrow \mathrm{V}^{X^{\mathrm{op}}}$. Moreover, for each $x \in X$ and $f \in \mathrm{V}^{X^{\mathrm{op}}}$, we have

$$
d(a(-, x), f)=f(x)
$$

Proof. Note that $d(a(-, x), f)=\bigwedge_{y} \operatorname{hom}(a(y, x), f(y)) \leq f(x)$. On the other hand, for each $y \in Y$,

$$
\begin{aligned}
a(y, x) \leq \operatorname{hom}(f(x), f(y)) & \Longleftrightarrow f(x) \otimes a(y, x) \leq f(y) \\
& \Longleftrightarrow f(x) \leq \operatorname{hom}(a(y, x), f(y)) .
\end{aligned}
$$

\subsection{Lawvere-complete V-categories.}

Definition. A $\mathrm{V}$-category $(X, a)$ is said to be Lawvere-complete if, for any $\mathrm{V}$-category $(Y, b)$, for every pair of adjoint bimodules

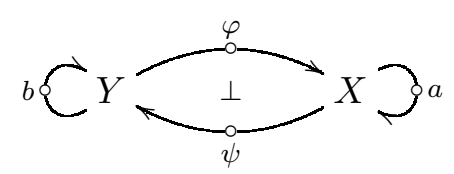

$\varphi$ is in the image of ()$_{*}: \mathrm{V}$-Cat $\rightarrow \mathrm{V}$-Mod, i.e. there exists a $\mathrm{V}$-functor $f:(X, a) \rightarrow(Y, b)$ such that $f_{*}=\varphi$ and $f^{*}=\psi$.

It is interesting to notice that, in order to check Lawvere completeness, we can restrict to the case $(Y, b)$ is the $\mathrm{V}$-category $(1, p)$, where $1=\{\star\}$ is a singleton and $p(\star, \star)=k$.

Proposition. For a $\mathrm{V}$-category $(X, a)$, the following conditions are equivalent:

(i) $(X, a)$ is Lawvere-complete;

(ii) for each pair of adjoint bimodules $(\varphi:(1, p) \multimap(X, a)) \dashv(\psi:(X, a) \multimap(1, p))$, there exists $a \mathrm{~V}$-functor $f:(1, p) \rightarrow(X, a)$ such that $\varphi=f_{*}$ and $\psi=f^{*}$ (in this situation we say that $f(\star)$ represents the adjunction $\varphi \dashv \psi)$.

Proof. It is a special case of Proposition 2.7. We omit the proof here because it follows, step by step, the proof of Proposition 2.7.

Theorem. The $\mathrm{V}$-category (V,hom) is Lawvere-complete.

Proof. Although this fact can be deduced from more general categorical results, we prefer to give here a direct proof, which provides guidance for the more general corresponding result for the $(\mathbb{T}, \bigvee)$-categorical structure of $\mathrm{V}$ we will study later.

Consider

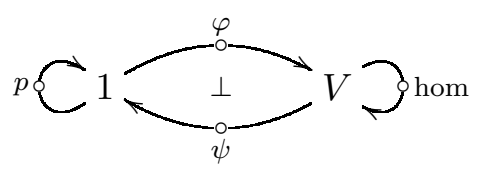

From the above theorem it follows that

$$
\begin{aligned}
\varphi \text { is a bimodule } & \Longleftrightarrow \varphi:(\mathrm{V}, \mathrm{hom}) \rightarrow(\mathrm{V}, \mathrm{hom}) \text { is a } \mathrm{V} \text {-functor } \\
& \Longleftrightarrow \forall u, v \in \mathrm{V} \operatorname{hom}(u, v) \leq \operatorname{hom}(\varphi(u), \varphi(v)) ;
\end{aligned}
$$




$$
\begin{aligned}
\psi \text { is a bimodule } & \Longleftrightarrow \psi:\left(\mathrm{V}, \operatorname{hom}^{\mathrm{op}}\right) \rightarrow(\mathrm{V}, \mathrm{hom}) \text { is a } \mathrm{V} \text {-functor } \\
& \Longleftrightarrow \forall u, v \in \mathrm{V} \operatorname{hom}(u, v) \leq \operatorname{hom}(\psi(v), \psi(u)) ;
\end{aligned}
$$

the conditions for the adjunction read as:

$$
\varphi \dashv \psi \Longleftrightarrow \forall u, v \in \mathrm{V} \quad \psi(u) \otimes \varphi(v) \leq \operatorname{hom}(u, v) \quad \& \quad k \leq \bigvee_{u \in V} \varphi(u) \otimes \psi(u)
$$

We will show that the adjunction $\varphi \dashv \psi$ is represented by $\psi(k)$, i.e, $\varphi(v)=\operatorname{hom}(\psi(k), v)$ and $\psi(v)=\operatorname{hom}(v, \psi(k))$, for every $v \in \mathrm{V}$. First we notice that from (3) it follows that $\psi(k) \otimes \varphi(v) \leq \operatorname{hom}(k, v)=v$, hence $\varphi(v) \leq \operatorname{hom}(\psi(k), v)$. Now the proof consists of checking three equalities:

(1st) $\psi(k)=\bigvee_{u \in \mathrm{V}} \psi(u) \otimes u$ :

It is immediate that $\psi(k)=\psi(k) \otimes k \leq \bigvee_{u \in \mathrm{V}} \psi(u) \otimes u$, and, moreover, for every $v \in \mathrm{V}$,

$$
\begin{aligned}
\psi(u) \otimes u=\psi(u) \otimes \operatorname{hom}(k, u) & \leq \psi(u) \otimes \operatorname{hom}(\psi(u), \psi(k)) \\
& \leq \psi(k) .
\end{aligned}
$$

(2nd) $\forall v \in \mathrm{V} \operatorname{hom}(v, \psi(k))=\bigvee_{u \in \mathrm{V}} \operatorname{hom}(v, u) \otimes \psi(u)$ :

To show " $\geq$ " we just observe that

$$
v \otimes(\operatorname{hom}(v, u) \otimes \psi(u)) \leq u \otimes \psi(u) \leq \psi(k)
$$

for " $\leq$ ", we have

$$
\begin{aligned}
\operatorname{hom}(v, \psi(k)) & \leq \operatorname{hom}(v, \psi(k)) \otimes \bigvee_{u \in V} \varphi(u) \otimes \psi(u) \\
& =\bigvee_{u \in V} \operatorname{hom}(v, \psi(k)) \otimes \varphi(u) \otimes \psi(u) \\
& \leq \bigvee_{u \in V} \operatorname{hom}(v, \psi(k)) \otimes \operatorname{hom}(\psi(k), u) \otimes \psi(u) \quad(\text { since } \varphi \leq \operatorname{hom}(\psi(k),-)) \\
& \leq \bigvee_{u \in V} \operatorname{hom}(v, u) \otimes \psi(u) .
\end{aligned}
$$

(3rd) Since $\psi=\psi \cdot$ hom we have $\forall v \in \mathrm{V} \psi(v)=\bigvee_{u \in V} \operatorname{hom}(v, u) \otimes \psi(u)$.

A new insight on Lawvere completeness for V-categories may be found in [25].

\section{Basic properties of $(\mathbb{T}, V)$-categories}

In the first part of this section we present the setting for the study of $(\mathbb{T}, V)$-categories, or (Eilenberg-Moore) lax algebras, that can be studied in more detail in [7, 10, 8]. 
2.1 $\mathbb{T}$ and its extension. Recall that a monad $\mathbb{T}=(T, e, m)$ on Set consists of a functor $T:$ Set $\rightarrow$ Set together with natural transformations $e: \operatorname{Id}_{\text {Set }} \rightarrow T$ (unit) and $m: T T \rightarrow T$ (multiplication) such that

$$
m \cdot T m=m \cdot m_{T} \quad \text { and } \quad m \cdot T e=1_{T}=m \cdot e_{T} .
$$

There are two trivial monads on Set, one sending all sets $X$ to the terminal set 1 , and the other with $T \varnothing=\varnothing$ and $T X=1$ for $X \neq \varnothing$. Any other monad is called non-trivial.

By a lax extension of a Set-monad $\mathbb{T}=(T, e, m)$ to $\mathrm{V}$-Mat we mean an extension of the endofunctor $T:$ Set $\rightarrow$ Set to $\mathrm{V}$-matrices acting on Set-maps as $T$ and satisfying

(a) $(T a)^{\circ}=T\left(a^{\circ}\right)\left(\right.$ and we write $\left.T a^{\circ}\right)$,

(b) $T b \cdot T a \leq T(b \cdot a)$,

(c) $a \leq a^{\prime} \Rightarrow T a \leq T a^{\prime}$,

(d) $e_{Y} \cdot a \leq T a \cdot e_{X}$,

(e) $m_{Y} \cdot T^{2} a \leq T a \cdot m_{X}$,

for all $a, a^{\prime}: X \mapsto Y$ and $b: Y \rightarrow Z$ 4. Note that we have automatically equality in (b) if $a=f$ is a Set-map. A Set-monad $\mathbb{T}=(T, e, m)$ admitting a lax extension to $\mathbf{V}$-Mat is called $\mathrm{V}$-admissible. Although $\mathbb{T}$ may have many lax extensions to $\mathrm{V}$-Mat, in the sequel we usually have a fixed extension in mind when talking about a $V$-admissible monad. Trivially, the identity $\operatorname{monad} \mathbb{1}=(\mathrm{Id}, 1,1)$ on Set can be extended to the identity monad on V-Mat. In [1] M. Barr shows how to extend Set-monads to Rel $=2$-Mat: first observe that each relation $r: X \rightarrow Y$ can be written as $r=p \cdot q^{\circ}$ where $q: R \rightarrow X$ and $p: R \rightarrow Y$ are the projection maps, then put $\operatorname{Tr}=T p \cdot T q^{\circ}$. All conditions above but the second one are satisfied, and this extension satisfies (b) if and only if the Set-functor $T$ has (BC) (that is, sends pullbacks to weak pullbacks). In [8] we showed how to make the step from Rel to $\mathrm{V}$-Mat, provided that in addition $\mathrm{V}$ is constructively completely distributive $(c c d)$. 5 . Given a monad $\mathbb{T}=(T, e, m)$ and a $\vee$-matrix $a: X \rightarrow Y$, we define relations $a_{v}: X \mapsto Y(v \in \mathrm{V})$ by $a_{v}(x, y)=$ true $\Longleftrightarrow a(x, y) \geq v$ and put, for $\mathfrak{x} \in T X$ and $\mathfrak{y} \in T Y$,

$$
T a(\mathfrak{x}, \mathfrak{y})=\bigvee\left\{v \in \mathrm{V} \mid T a_{v}(\mathfrak{x}, \mathfrak{y})=\text { true }\right\}
$$

Then the formula above defines an extension of $T:$ Set $\rightarrow$ Set provided that either $k=\top$ or $T \varnothing=\varnothing$. Moreover, all five conditions above are satisfied. In addition we have

(f) $T b \cdot T a=T(b \cdot a)$ provided that $\otimes=\wedge$,

(g) $T g \cdot T a=T(g \cdot a)$,

for all $\mathrm{V}$-matrices $a: X \longrightarrow Y$ and $b: Y \rightarrow Z$ and all maps $g: Y \rightarrow Z$. In some occasions we will need that the (Set-based) natural transformation $m: T T \rightarrow T$ has (BC) (that is, each naturality square is a weak pullback); this guarantees that $m$ is also a (strict) natural transformation for the extension of $T$ to $\mathrm{V}$-Mat described above.

\footnotetext{
${ }^{4}$ The conditions for our extension are stronger than Seal's in 24 .

${ }^{5}$ Recall that a lattice $Y$ is (ccd) if $\bigvee: 2^{Y^{\mathrm{op}}} \rightarrow Y$ has a left adjoint; for more details see [27.
} 
$2.2(\mathbb{T}, \mathrm{V})$-categories. Let $\mathbb{T}=(T, e, m)$ be a $\mathrm{V}$-admissible monad. $\mathrm{A}(\mathbb{T}, \mathrm{V})$-category is a pair $(X, a)$ consisting of a set $X$ and a $\mathrm{V}$-matrix $a: T X \mapsto X$ such that:

$$
1_{X} \leq a \cdot e_{X} \text { and } a \cdot T a \leq a \cdot m_{X}
$$

that is, the map $a: T X \times X \rightarrow \mathrm{V}$ satisfies the conditions:

(R) for each $x \in X, k \leq a\left(e_{X}(x), x\right)$;

(T) for each $\mathfrak{X} \in T^{2} X, \mathfrak{x} \in T X, x \in X, T a(\mathfrak{X}, \mathfrak{x}) \otimes a(\mathfrak{x}, x) \leq a\left(m_{X}(\mathfrak{X}), x\right)$.

Given $(\mathbb{T}, \mathrm{V})$-categories $(X, a)$ and $(Y, b)$, a $(\mathbb{T}, \bigvee)$-functor $f:(X, a) \rightarrow(Y, b)$ is a map $f:$ $X \rightarrow Y$ such that, for each $\mathfrak{x} \in T X$ and $x \in X, a(\mathfrak{x}, x) \leq b(T f(\mathfrak{x}), f(x))$. (T,, $\mathrm{V})$-categories and $(\mathbb{T}, \bigvee)$-functors are the objects and morphisms of the category $(\mathbb{T}, \bigvee)$-Cat.

Note that each Eilenberg-Moore algebra for $\mathbb{T}$ can be viewed as a $(\mathbb{T}, V)$-category; in fact, we have an embedding

$$
\text { Set }^{\mathbb{T}} \hookrightarrow(\mathbb{T}, \mathrm{V}) \text {-Cat. }
$$

In particular, for each set $X$ we have the $(\mathbb{T}, \mathrm{V})$-category $\left(T X, m_{X}\right)$ which we denote by $|X|$.

Obviously, each $\vee$-category is a $(\mathbb{T}, V)$-category for $\mathbb{T}=\mathbb{1}$ the identity monad "identically" extended to $\mathrm{V}$-Mat. A further class of interesting examples involves the ultrafilter monad $\mathbb{U}=$ $(U, e, m)$. The extension of $U:$ Set $\rightarrow$ Set to V-Mat of 2.1 can be equivalently described by

$$
U r(\mathfrak{x}, \mathfrak{y})=\bigwedge_{(A \in \mathfrak{x}, B \in \mathfrak{y})} \bigvee_{(x \in A, y \in B)} r(x, y),
$$

for all $r: X \mapsto Y$ in $\mathrm{V}$-Mat, $\mathfrak{x} \in T X$ and $\mathfrak{y} \in T Y$. The main result of [1] states that (U⿺,2)-Cat $\cong$ Top. In [7] it is shown that $\left(\mathbb{U}, P_{+}\right)$-Cat $\cong A p p$, the category of approach spaces and nonexpansive maps (see [19] for details.)

2.3 The dual $(\mathbb{T}, V)$-category. We have the canonical forgetful functor

$$
\begin{aligned}
E:(\mathbb{T}, \mathrm{V})-\text { Cat } & \rightarrow \text { V-Cat, } \\
(X, a) & \mapsto\left(X, a \cdot e_{X}\right)
\end{aligned}
$$

with left adjoint

$$
\begin{aligned}
E^{\circ}: \mathrm{V}-\text { Cat } & \rightarrow(\mathbb{T}, \mathrm{V}) \text {-Cat. } \\
(X, a) & \mapsto\left(X, e_{X}^{\circ} \cdot T a\right)
\end{aligned}
$$

Furthermore, (the extension of) $T$ induces an endofunctor

$$
\begin{aligned}
T: V \text {-Cat } & \rightarrow V \text {-Cat. } \\
(X, a) & \mapsto(T X, T a)
\end{aligned}
$$

If $m$ is a (strict) natural transformation, we can represent this functor as the composite

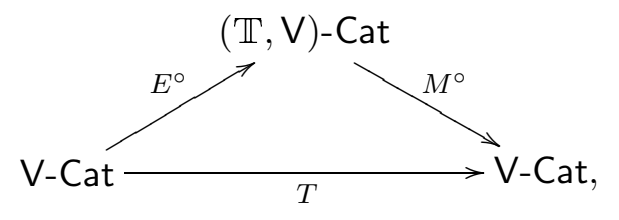


where $M^{\circ}:(\mathbb{T}, \mathrm{V})$-Cat $\rightarrow \mathrm{V}$-Cat is given by $(X, a) \mapsto\left(T X, T a \cdot m_{X}^{\circ}\right)$. In fact, given a $\mathrm{V}$-category $(X, a)$, we have

$$
T\left(e_{X}^{\circ} \cdot T a\right) \cdot m_{X}^{\circ}=T e_{X}^{\circ} \cdot T^{2} a \cdot m_{X}^{\circ}=T e_{X}^{\circ} \cdot m_{X}^{\circ} \cdot T a=T a .
$$

The functors $M^{\circ}$ and $E^{\circ}$ are the keys to define the dual $(\mathbb{T}, \mathrm{V})$-category $X^{\text {op }}$ of a $(\mathbb{T}, \bigvee)$-category $X=(X, a)$ : we put $X^{\mathrm{op}}=E^{\circ}\left(M^{\circ}(X)^{\mathrm{op}}\right)$. We point out that if $X$ is a $\mathrm{V}$-category interpreted as a $(\mathbb{T}, \mathrm{V})$-category, i.e. $X=\left(X, e_{X}^{\circ} \cdot T a\right)$ for a given $\mathrm{V}$-category structure $a: X \rightarrow X$, then

$$
X^{\mathrm{op}}=E^{\circ}\left(M^{\circ}\left(E^{\circ}(X, a)\right)^{\mathrm{op}}\right)=E^{\circ}\left((T X, T a)^{\mathrm{op}}\right),
$$

that is, $X^{\mathrm{op}}$ is the dual - as a V-category - of $T(X, a)$.

Our Theorem 3.3 shows that this is indeed a reasonable definition.

Finally, for later use we record the following

Lemma. Let $(X, a)$ be a $\mathrm{V}$-category and $(X, \alpha)$ be a $\mathbb{T}$-algebra. Then $(X, a \cdot \alpha)$ is a $(\mathbb{T}, \mathrm{V})$ category if and only if $\alpha:(T X, T a) \rightarrow(X, a)$ is a $\mathrm{V}$-functor.

Proof. First we remark that from $1_{X} \leq a$ and $1_{X}=\alpha \cdot e_{X}$ it follows that $1_{X} \leq(a \cdot \alpha) \cdot e_{X}$, that is $a \cdot \alpha$ always fulfils the reflexivity axiom. Now, if $\alpha$ is a $\mathrm{V}$-functor, i.e. $\alpha \cdot T a \leq a \cdot \alpha$, then

$$
(a \cdot \alpha) \cdot T(a \cdot \alpha)=a \cdot \alpha \cdot T a \cdot T \alpha \leq a \cdot a \cdot \alpha \cdot T \alpha \leq(a \cdot \alpha) \cdot m_{X} .
$$

Conversely, if $a \cdot \alpha$ is a $(\mathbb{T}, \mathrm{V})$-categorical structure, then

$$
\alpha \cdot T a=\alpha \cdot T a \cdot T \alpha \cdot T e_{X} \leq a \cdot \alpha \cdot T a \cdot T \alpha \cdot T e_{X} \leq a \cdot \alpha \cdot m_{X} \cdot T e_{X}=a \cdot \alpha .
$$

2.4 Kleisli composition. Many notions and techniques can be transported from $\mathrm{V}$-Cat to $(\mathbb{T}, \mathrm{V})$-Cat by formally replacing composition of $\mathrm{V}$-matrices by Kleisli composition (see [15]) defined as

$$
b * a:=b \cdot T a \cdot m_{X}^{\circ},
$$

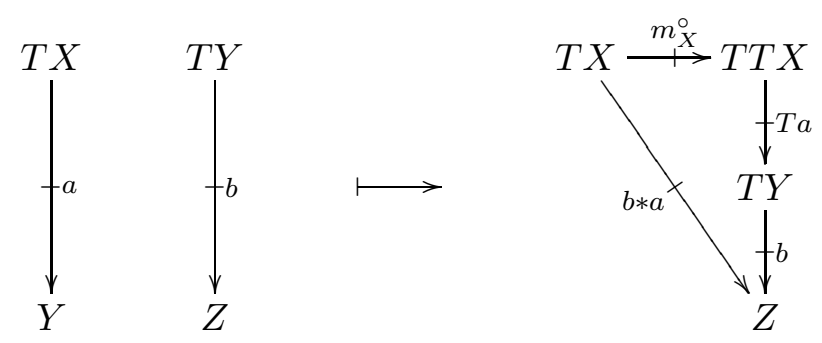

for all $a: T X \mapsto Y$ and $b: T Y \rightarrow Z$ in $\mathrm{V}$-Mat. The matrix $e_{X}^{\circ}: T X \mapsto X$ acts as a lax identity for this composition, in the following sense:

$$
a * e_{X}^{\circ}=a \quad \text { and } \quad e_{X}^{\circ} * b \geq b,
$$

for $a: T X \mapsto Y$ and $b: T Y \mapsto X$. Moreover,

$$
c *(b * a) \leq(c * b) * a
$$

if $T: \mathrm{V}$-Mat $\rightarrow$ V-Mat preserves composition, and

$$
c *(b * a) \geq(c * b) * a
$$

if $m: T T \rightarrow T$ is a (strict) natural transformation. 
2.5 ( $\mathbb{T}, \mathrm{V})$-bimodules. Given $(\mathbb{T}, \mathrm{V})$-categories $(X, a)$ and $(Y, b)$, a $(\mathbb{T}, \mathrm{V})$-bimodule (or simply a bimodule $):(X, a) \rightarrow(Y, b)$ is a $\vee$-matrix $\psi: T X \mapsto Y$ such that $\psi * a \leq \psi$ and $b * \psi \leq \psi$. This means that $\psi \cdot T a \cdot m_{X}^{\circ} \leq \psi$ and $b \cdot T \psi \cdot m_{X}^{\circ} \leq \psi$; that is, for $\mathfrak{X} \in T^{2} X, \mathfrak{x} \in T X, \mathfrak{y} \in T Y$ and $y \in Y$,

$$
\begin{aligned}
& T a(\mathfrak{X}, \mathfrak{x}) \otimes \psi(\mathfrak{x}, y) \leq \psi\left(m_{X}(\mathfrak{X}), y\right), \\
& T \psi(\mathfrak{X}, \mathfrak{y}) \otimes b(\mathfrak{y}, y) \leq \psi\left(m_{X}(\mathfrak{X}), y\right) .
\end{aligned}
$$

Whenever the Kleisli composition is associative (in particular if $T: \mathrm{V}$-Mat $\rightarrow \mathrm{V}$-Mat is a functor and $m$ is a natural transformation: see [15]), bimodules compose. The identities for the composition law are again the $(\mathbb{T}, V)$-categorical structures, and we can consider the category $(\mathbb{T}, \mathrm{V})$-Mod of $(\mathbb{T}, \mathrm{V})$-categories and $(\mathbb{T}, \mathrm{V})$-bimodules.

2.6 ( $\mathbb{T}, \mathrm{V})$-functors as $(\mathbb{T}, \mathrm{V})$-bimodules. Analogously to the situation in $\mathrm{V}$-categories, each $(\mathbb{T}, \bigvee)$-functor $f:(X, a) \rightarrow(Y, b)$ defines a pair of bimodules $f_{*}:(X, a) \rightarrow(Y, b)$ and $f^{*}:(Y, b) \rightarrow(X, a)$ as indicated in the following diagram

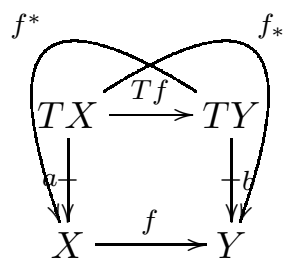

that is, $f_{*}:=b \cdot T f$ and $f^{*}:=f^{\circ} \cdot b$. In fact, the following assertions are equivalent for $(\mathbb{T}, \mathrm{V})$ categories $(X, a)$ and $(Y, b)$ and a function $f: X \rightarrow Y$.

(i) $f:(X, a) \rightarrow(Y, b)$ is a $(\mathbb{T}, \vee)$-functor.

(ii) $f_{*}:(X, a) \rightarrow(Y, b)$ is a $(\mathbb{T}, \mathrm{V})$-bimodule.

(iii) $f^{*}:(Y, b) \multimap(X, a)$ is a $(\mathbb{T}, \mathrm{V})$-bimodule.

We point out that, although in general bimodules do not compose, if $f:(X, a) \rightarrow(Y, b)$ is a functor, then, for any bimodules $\varphi:(Y, b) \rightarrow(Z, c)$ and $\psi:(Z, c) \rightarrow(Y, b)$,

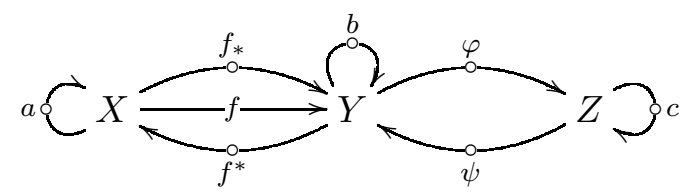

$\varphi * f_{*}$ and $f^{*} * \psi$ are bimodules, as we show next. First note that

$$
\varphi * f_{*}=\varphi \cdot T f \quad \text { and } \quad f^{*} * \psi=f^{\circ} \cdot \psi
$$

The latter equality follows from

$$
f^{*} * \psi=f^{\circ} \cdot b \cdot T \psi \cdot m_{Z}^{\circ}=f^{\circ} \cdot \psi,
$$


and $\mathrm{V}$-functoriality of $f$ implies

$$
\begin{array}{rlrl}
\varphi * f_{*}=\varphi *(b \cdot T f) & =\varphi \cdot T(b \cdot T f) \cdot m_{X}^{\circ} & & \\
& \geq \varphi \cdot T(f \cdot a) \cdot m_{X}^{\circ} & & \text { (by functoriality of } f \text { ) } \\
& \geq \varphi \cdot T f \cdot T a \cdot m_{X}^{\circ} & \\
& \geq \varphi \cdot T f \cdot T a \cdot T e_{X} & & \text { (since } \left.m_{X}^{\circ} \geq T e_{X}\right) \\
& \geq \varphi \cdot T f, & & \left(\text { since } a \cdot e_{X} \geq 1_{X}\right)
\end{array}
$$

whereby $\varphi$ bimodule gives us

$$
\varphi * f_{*}=\varphi *(b \cdot T f)=\varphi \cdot T b \cdot T^{2} f \cdot m_{X}^{\circ} \leq \varphi \cdot T b \cdot m_{Y}^{\circ} \cdot T f=\varphi \cdot T f .
$$

The bimodule properties of $\varphi * f_{*}$ and $f^{*} * \psi$ follow now from

$$
\begin{aligned}
& c *\left(\varphi * f_{*}\right)=c *(\varphi \cdot T f) \leq(c * \varphi) \cdot T f=\varphi * f_{*}, \\
& \left(\varphi * f_{*}\right) * a=\varphi \cdot T f \cdot T a \cdot m_{X}^{\circ} \leq \varphi \cdot T b \cdot T^{2} f \cdot m_{X}^{\circ} \leq \varphi \cdot T b \cdot m_{Y}^{\circ} \cdot T f=\varphi * f_{*}, \\
& a *\left(f^{*} * \psi\right)=a \cdot T\left(f^{\circ} \cdot \psi\right) \cdot m_{Z}^{\circ}=a \cdot T f^{\circ} \cdot T \psi \cdot m_{Z}^{\circ} \leq f^{\circ} \cdot b \cdot T \psi \cdot m_{Z}^{\circ}=f^{\circ} \cdot \psi=f^{*} * \psi, \\
& \left(f^{*} * \psi\right) * c=f^{\circ} \cdot \psi \cdot T c \cdot m_{Z}^{\circ}=f^{\circ} \cdot(\psi * c)=f^{\circ} \cdot \psi=f^{*} * \psi .
\end{aligned}
$$

Therefore we can define the "whiskering" functors

$$
\begin{aligned}
-* f_{*}:(\mathbb{T}, \mathrm{V})-\operatorname{Mod}(Y, Z) & \longrightarrow(\mathbb{T}, \mathrm{V})-\operatorname{Mod}(X, Z), \text { and } \\
\varphi & \longmapsto \varphi \cdot T f \\
f^{*} *:(\mathbb{T}, \mathrm{V})-\operatorname{Mod}(Z, Y) & \longrightarrow(\mathbb{T}, \mathrm{V})-\operatorname{Mod}(Z, X) \\
\psi & \longmapsto f^{\circ} \cdot \psi
\end{aligned}
$$

Moreover, given a pair of adjoint bimodules $(\varphi:(Y, b) \multimap(Z, c)) \dashv(\psi:(Z, c) \rightarrow(Y, b))$, we have

$$
\varphi * f_{*} \dashv f^{*} * \psi
$$

provided that the diagram

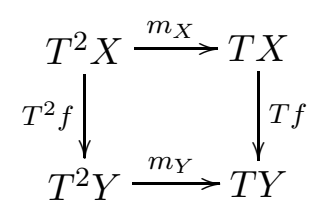

satisfies $(\mathrm{BC}):\left(\varphi * f_{*}\right) *\left(f^{*} * \psi\right) \leq c$ is always true, since

$$
(\varphi \cdot T f) *\left(f^{\circ} \cdot \psi\right)=\varphi \cdot T f \cdot T f^{\circ} \cdot T \psi \cdot m_{Z}^{\circ} \leq \varphi \cdot T \psi \cdot m_{Z}^{\circ}=\varphi * \psi \leq c,
$$

while to conclude that $a \leq\left(f^{*} * \psi\right) *\left(\varphi * f_{*}\right)$ we need the condition above:

$$
a \leq f^{\circ} \cdot b \cdot T f \leq f^{\circ} \cdot \psi \cdot T \varphi \cdot m_{Y}^{\circ} \cdot T f=f^{\circ} \cdot \psi \cdot T \varphi \cdot T^{2} f \cdot m_{X}^{\circ}=\left(f^{\circ} \cdot \psi\right) *(\varphi \cdot T f) .
$$

\subsection{Lawvere-complete $(\mathbb{T}, \bigvee)$-categories.}


Definition. A $(\mathbb{T}, \mathrm{V})$-category $(X, a)$ is called Lawvere-complete if, for each $(\mathbb{T}, \mathrm{V})$-category $(Y, b)$ and each pair of adjoint bimodules

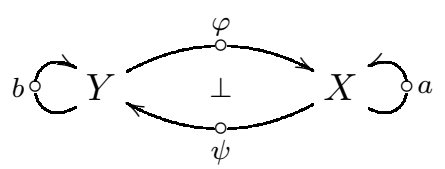

there exists a functor $f:(Y, b) \rightarrow(X, a)$ such that $f_{*}=\varphi$ and $f^{*}=\psi$.

Analogously to the $\mathrm{V}$-categorical case, Lawvere completeness is fully tested by left adjoint bimodules with domain $(1, p)$, where $p=e_{1}^{\circ}$, hence $p(\dot{\star}, \star)=k$ and $p(\mathfrak{x}, \star)=\perp$ for $\mathfrak{x} \neq \dot{\star}$ in $T 1$.

Proposition. Assume that either $T 1=1$ or that the (Set-based) natural transformation $m$ satisfies $(\mathrm{BC})$. Then, for a $(\mathbb{T}, V)$-category $(X, a)$, the following conditions are equivalent:

(i) $(X, a)$ is Lawvere-complete;

(ii) each pair of adjoint bimodules

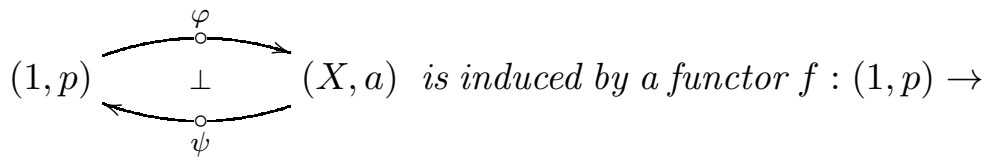
$(X, a)$

(iii) each pair of adjoint bimodules

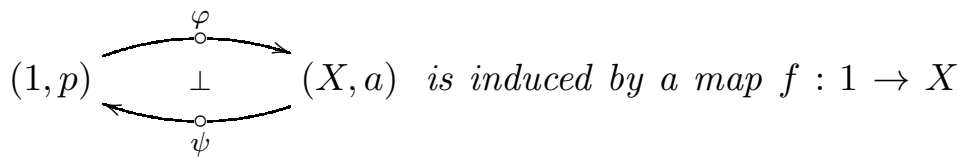
(so that $\varphi=a \cdot T f$ and $\psi=f^{\circ} \cdot a$ ).

Proof. (iii) $\Rightarrow(\mathrm{i})$ : Let $(Y, b) \underset{+}{+}(X, a)$ be a pair of adjoint bimodules. For each $y \in Y$, let $g_{y}:(1, p) \rightarrow(Y, b)$ be the functor that picks $y$. This functor induces a pair of adjoint bimodules $\left(g_{y}\right)_{*} \dashv\left(g_{y}\right)^{*}$, whence we have

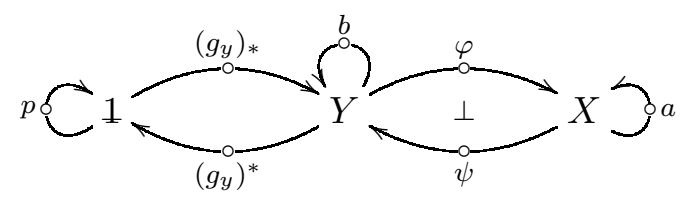

If $m$ satisfies (BC), we know already that $\varphi_{y} \dashv \psi_{y}$, where $\varphi_{y}=\varphi *\left(g_{y}\right)_{*}=\varphi \cdot T g_{y}$ and $\psi_{y}=\left(g_{y}\right)^{*} * \psi=g_{y}^{\circ} \cdot \psi$. The same happens whenever $T 1=1$, as it is easily checked. By hypothesis, there exists a map $f_{y}: 1 \rightarrow X$ such that $\varphi_{y}=b \cdot T f_{y}$ and $\psi_{y}=f_{y}^{\circ} \cdot b$. Gluing together the maps $\left(f_{y}\right)_{y \in Y}$ we obtain a map $f: Y \rightarrow X$. Then, for $\mathfrak{x} \in T X$ and $y \in Y$,

$$
\psi(\mathfrak{x}, y)=\psi_{y}(\mathfrak{x}, \star)=f_{y}^{\circ} \cdot a(\mathfrak{x}, \star)=a\left(\mathfrak{x}, f_{y}(\star)\right)=a(\mathfrak{x}, f(y))
$$

that is, $\psi=f^{*}=f^{\circ} \cdot a$. We can show now that $f$ is necessarily a functor:

$$
b \cdot T f^{\circ} \leq b \cdot T f^{\circ} \cdot T a \cdot T e_{X} \leq b \cdot T f^{\circ} \cdot T a \cdot m_{X}^{\circ}=b * \psi \leq \psi=f^{\circ} \cdot a .
$$

This concludes the proof since, by unicity of adjoints, $\varphi$ is necessarily $f_{*}$. 


\section{$3 \quad \vee$ as a $(\mathbb{T}, V)$-category}

3.1 The $\mathbb{T}$-algebra structure of $\mathrm{V}$. Our next goal is to explore the notions introduced in the previous section. In particular we are aiming for results which extend known facts about V-categories (as Theorem 1.5 or Theorem 1.6). To do so, from now on we will always assume that the extension $T: \mathrm{V}-\mathrm{Mat} \rightarrow \mathrm{V}$-Mat is constructed as in [8] and consequently we assume $\checkmark$ to be constructively completely distributive. Furthermore, we assume that $\mathbb{T}=(T, e, m)$ is non-trivial and that $T$ and $m$ satisfy $(B C)$.

Under these conditions, as Manes essentially showed in [21],

$$
\begin{aligned}
\xi: T \mathrm{~V} & \longrightarrow \mathrm{V} \\
\mathfrak{x} & \longmapsto \bigvee\{v \in \mathrm{V} \mid \mathfrak{x} \in T(\uparrow v)\}
\end{aligned}
$$

is a $\mathbb{T}$-algebra structure on $\mathrm{V}$, where $\uparrow v=\{u \in \mathrm{V} \mid v \leq u\}$.

There is an interesting link between this $\mathbb{T}$-algebra structure and the image under the lax functor $T: \mathrm{V}$-Mat $\rightarrow \mathrm{V}$-Mat of the identity $1_{\mathrm{V}}: \mathrm{V} \rightarrow \mathrm{V}$ considered as a matrix $i: 1 \longrightarrow \mathrm{V}$, with $i(\star, v)=v$. Let us compute $T i: T 1 \longmapsto T \mathrm{~V}$. We consider, for each $v \in \mathrm{V}$, the relation

$$
\begin{aligned}
i_{v}: 1 \times V & \longrightarrow 2 \\
(\star, u) & \longmapsto \begin{cases}\text { true } & \text { if } v \leq u, \\
\text { false } & \text { elsewhere }\end{cases}
\end{aligned}
$$

hence the diagram

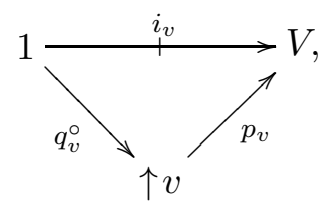

commutes where $p_{v}$ and $q_{v}$ are the projections. Now, for each $\mathfrak{x} \in T 1$ and $\mathfrak{y} \in T \vee$,

$$
\begin{aligned}
T i(\mathfrak{x}, \mathfrak{y}) & =\bigvee\left\{v \in \mathrm{V} \mid T\left(i_{v}\right)(\mathfrak{x}, \mathfrak{y})=\text { true }\right\} \\
& =\bigvee\left\{v \in \mathrm{V} \mid T p_{v} T q_{v}^{\circ}(\mathfrak{x}, \mathfrak{y})=\text { true }\right\} \\
& =\bigvee\left\{v \in \mathrm{V} \mid \exists \mathfrak{z} \in \uparrow v: T q_{v}(\mathfrak{z})=\mathfrak{x} \text { and } T p_{v}(\mathfrak{z})=\mathfrak{y}\right\}
\end{aligned}
$$

hence, since $T$ preserves injections and considering $T p_{v}$ as an inclusion, we can write

$$
T i(\mathfrak{x}, \mathfrak{y})=\bigvee\left\{v \in \mathrm{V} \mid \mathfrak{y} \in T(\uparrow v) \text { and } T q_{v}(\mathfrak{y})=\mathfrak{x}\right\} \leq \xi(\mathfrak{y})
$$

by definition of $\xi$. In particular, if $\mathfrak{x}=T q(\mathfrak{y})$, for $q: \mathrm{V} \rightarrow 1$, then $\operatorname{Ti}(\mathfrak{x}, \mathfrak{y})=\xi(\mathfrak{y})$. Whenever $T 1=1, T q(\mathfrak{y})=\dot{\star}$ for every $\mathfrak{y} \in T \vee$, whence

$$
T i(\dot{\star}, \mathfrak{y})=\bigvee\{v \in \mathrm{V} \mid \mathfrak{y} \in T(\uparrow v)\}=\xi(\mathfrak{y}) .
$$

This link between the extension of $T$ and the $\mathbb{T}$-algebra structure $\xi$ is more general. Whenever necessary, in the sequel we denote the Set-endofunctor $T$ by $T_{\mathrm{o}}$, and keep $T$ for its extension to $\mathrm{V}$-Mat. Each $\mathrm{V}$-matrix $r: X \mapsto Y$ can be considered also as a map $r: X \times Y \rightarrow \mathrm{V}$. The interplay between $T_{\mathrm{o}} r$ and $T r$ is stated in the following result, whose proof is straightforward. 
Proposition. For any V-matrix $r: X \rightarrow Y$, each $\mathfrak{x} \in T X$ and $\mathfrak{y} \in T Y$,

$$
\operatorname{Tr}(\mathfrak{x}, \mathfrak{y})=\bigvee_{\mathfrak{w}:} \xi \cdot T_{\mathrm{o}} r(\mathfrak{w}),
$$

that is the following diagram

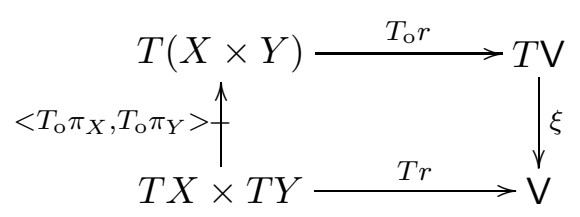

commutes.

Remark. Besides being the structure map of an Eilenberg-Moore algebra, $\xi: T \mathrm{~V} \rightarrow \mathrm{V}$ satisfies also the inequalities

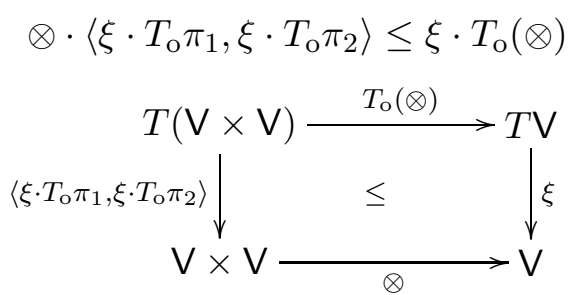

and

$$
k \cdot \leq \xi \cdot T_{\mathrm{o}} k
$$

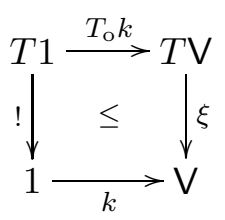

Recall that we assume $T f=T_{\mathrm{o}} f$ for each Set-map $f: X \rightarrow Y$; this condition requires and implies equality in the latter inequality (see [16]).

3.2 The canonical $(\mathbb{T}, \mathrm{V})$-categorical structure of $\mathrm{V}$. The composition of the natural

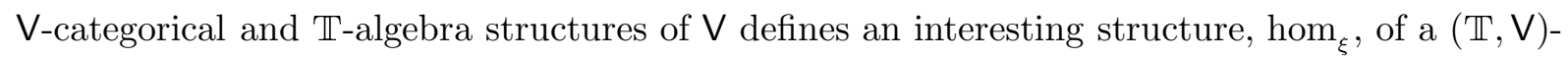
category on $\mathrm{V}$

$$
T \mathrm{~V} \stackrel{\operatorname{hom}_{\xi}}{\rightarrow} \mathrm{V}=(T \mathrm{~V} \stackrel{\xi}{\rightarrow} \mathrm{V} \stackrel{\text { hom }}{\rightarrow} \mathrm{V})
$$

as we show next.

Proposition. $\xi:(T \vee, T$ hom $) \rightarrow(\mathrm{V}$, hom $)$ is a $\vee$-functor.

Proof. We have to show that $\xi \cdot T$ hom $\leq$ hom $\cdot \xi$, or, equivalently, $T$ hom $\leq \xi^{\circ} \cdot$ hom $\cdot \xi$. This means that, for $\mathfrak{x}, \mathfrak{y} \in T \mathrm{~V}$,

$$
T \operatorname{hom}(\mathfrak{x}, \mathfrak{y}) \leq \operatorname{hom}(\xi(\mathfrak{x}), \xi(\mathfrak{y})) .
$$

We consider again the matrix $i: 1 \nrightarrow V$, and compute $1 \stackrel{i}{\rightarrow} \mathrm{V} \stackrel{\text { hom }}{\rightarrow} \mathrm{V}$ :

$$
\operatorname{hom} \cdot i(\star, v)=\bigvee_{u \in V} i(\star, u) \otimes \operatorname{hom}(u, v)=\bigvee_{u \in \bigvee} u \otimes \operatorname{hom}(u, v) \leq v ;
$$

that is hom $\cdot i \leq i$. Hence $T \operatorname{hom} \cdot T i \leq T(\operatorname{hom} \cdot i) \leq T i$, and so, for $\mathfrak{x}, \mathfrak{y} \in T \vee$ and $\mathfrak{z}=T q(\mathfrak{x})$ as in Section 3.1, we have

$$
\xi(\mathfrak{x}) \otimes T \operatorname{hom}(\mathfrak{x}, \mathfrak{y})=T i(\mathfrak{z}, \mathfrak{x}) \otimes T \operatorname{hom}(\mathfrak{x}, \mathfrak{y}) \leq T i(\mathfrak{z}, \mathfrak{y}) \leq \xi(\mathfrak{y}),
$$

and therefore

$$
T \operatorname{hom}(\mathfrak{x}, \mathfrak{y}) \leq \operatorname{hom}(\xi(\mathfrak{x}), \xi(\mathfrak{y}))
$$

as claimed. 
Corollary. $\left(\mathrm{V}, \mathrm{hom}_{\xi}\right)$ is a $(\mathbb{T}, \mathrm{V})$-category.

Proof. Follows from the proposition above and Lemma 2.2 ,

3.3 The tensor product. The tensor product in $\mathrm{V}$ defines in a natural way a (not necessarily closed) product structure in $(\mathbb{T}, \mathrm{V})$-Cat. Given $(\mathbb{T}, \mathrm{V})$-categories $X=(X, a)$ and $Y=(Y, b)$, we put $X \otimes Y=(X \times Y, a \otimes b)$ where $a \otimes b(\mathfrak{w},(x, y))=a\left(T \pi_{X}(\mathfrak{w}), x\right) \otimes b\left(T \pi_{Y}(\mathfrak{w}), y\right)$ for all $\mathfrak{w} \in T(X \times Y), x \in X$ and $y \in Y$. One easily verifies reflexivity of $a \otimes b$, while transitivity holds if and only if $\otimes \cdot\left\langle\xi \cdot T_{\mathrm{o}} \pi_{1}, \xi \cdot T_{\mathrm{o}} \pi_{2}\right\rangle=\xi \cdot T_{\mathrm{o}}(\otimes)$ (see Remark 3.1 and [16]) which we assume from now on. We remark that this condition guarantees that $\mathbb{T}$ is a (lax) Hopf monad on $\mathrm{V}-\mathrm{Mat}$ (see [22]) where the tensor product in $\mathrm{V}$ is naturally extended to $\mathrm{V}$-Mat. However, we will not develop this aspect here.

It is well-known that in general the functor $X \otimes \otimes_{-}:(\mathbb{T}, \mathrm{V})$-Cat $\rightarrow(\mathbb{T}, \mathrm{V})$-Cat has no right adjoint as, for example, Top is not Cartesian closed. The problem of characterising those $(\mathbb{T}, V)$ categories $X=(X, a)$ such that tensoring with $X$ has a right adjoint is studied in [16].

Theorem. Let $m$ be a natural transformation. For $(\mathbb{T}, \mathrm{V})$-categories $(X, a)$ and $(Y, b)$ and a $\mathrm{V}$-matrix $\psi: T X \mapsto Y$, the following assertions are equivalent.

(i) $\psi:(X, a) \rightarrow(Y, b)$ is a $(\mathbb{T}, \mathrm{V})$-bimodule.

(ii) Both $\psi:|X| \otimes Y \rightarrow \vee$ and $\psi: X^{\mathrm{op}} \otimes Y \rightarrow \mathrm{\vee}$ are $(\mathbb{T}, \mathrm{V})$-functors.

Proof. Assume that $\psi:(X, a) \multimap(Y, b)$ is a $(\mathbb{T}, \mathcal{V})$-bimodule. First observe that, for $\mathfrak{W} \in$ $T(T X \times Y)$,

$$
\xi \cdot T_{\mathrm{o}} \psi(\mathfrak{W}) \leq T \psi\left(T_{\mathrm{o}} \pi_{T X}(\mathfrak{W}), T_{\mathrm{o}} \pi_{Y}(\mathfrak{W})\right) .
$$

Let $\mathfrak{W} \in T(T X \times Y), \mathfrak{x} \in T X$ and $y \in Y$. To see that $\psi:|X| \otimes Y \rightarrow \vee$ is a (T, $\mathbb{V})$-functor, note that the structure $c$ on $|X| \otimes Y$ is given by

$$
c(\mathfrak{W},(\mathfrak{x}, y))= \begin{cases}\perp & \text { if } \mathfrak{x} \neq m_{X}\left(T_{\mathrm{o}} \pi_{T X}(\mathfrak{W})\right), \\ b\left(T \pi_{Y}(\mathfrak{W}), y\right) & \text { if } \mathfrak{x}=m_{X}\left(T_{\mathrm{o}} \pi_{T X}(\mathfrak{W})\right) .\end{cases}
$$

Assume $\mathfrak{x}=m_{X}\left(T_{\mathrm{o}} \pi_{T X}(\mathfrak{W})\right)$. Since

$$
b\left(T_{\mathrm{o}} \pi_{Y}(\mathfrak{W}), y\right) \leq \operatorname{hom}\left(\xi \cdot T_{\mathrm{o}} \psi(\mathfrak{W}), \psi(\mathfrak{x}, y)\right)
$$

is equivalent to

$$
\xi \cdot T_{\mathrm{o}} \psi(\mathfrak{W}) \otimes b\left(T_{\mathrm{o}} \pi_{Y}(\mathfrak{W}), y\right) \leq \psi(\mathfrak{x}, y),
$$

the assertion follows at once. We show now that $\psi: X^{\mathrm{op}} \otimes Y \rightarrow \mathrm{V}$ is a $(\mathbb{T}, \mathrm{V})$-functor. As above we have that (with $a^{\mathrm{op}}=e_{T X}^{\circ} \cdot T m_{X} \cdot T^{2} a^{\circ}$ the structure on $X^{\mathrm{op}}$ )

$$
a^{\mathrm{op}}\left(T_{\mathrm{o}} \pi_{T X}(\mathfrak{W}), \mathfrak{x}\right) \otimes b\left(T_{\mathrm{o}} \pi_{Y}(\mathfrak{W}), y\right) \leq \operatorname{hom}\left(\xi \cdot T_{o} \psi(\mathfrak{W}), \psi(\mathfrak{x}, y)\right)
$$

is equivalent to

$$
\xi \cdot T_{\mathrm{o}} \psi(\mathfrak{W}) \otimes a^{\mathrm{op}}\left(T_{\mathrm{o}} \pi_{T X}(\mathfrak{W}), \mathfrak{x}\right) \otimes b\left(T_{\mathrm{o}} \pi_{Y}(\mathfrak{W}), y\right) \leq \psi(\mathfrak{x}, y)
$$


Now

$$
\begin{aligned}
\xi \cdot T_{\mathrm{o}} \psi(\mathfrak{W}) & \otimes a^{\mathrm{op}}\left(T_{\mathrm{o}} \pi_{T X}(\mathfrak{W}), \mathfrak{x}\right) \otimes b\left(T_{\mathrm{o}} \pi_{Y}(\mathfrak{W}), y\right) \\
& \leq T^{2} a \cdot T m_{X}^{\circ} \cdot e_{T X}\left(\mathfrak{x}, T_{\mathrm{o}} \pi_{T X}(\mathfrak{W})\right) \otimes T \psi\left(T_{\mathrm{o}} \pi_{T X}(\mathfrak{W}), T_{\mathrm{o}} \pi_{Y}(\mathfrak{W})\right) \otimes b\left(T_{\mathrm{o}} \pi_{Y}(\mathfrak{W}), y\right) \\
& \leq b \cdot T \psi \cdot T^{2} a \cdot T m_{X}^{\circ} \cdot m_{X}^{\circ}(\mathfrak{x}, y) \\
& \leq b \cdot T \psi \cdot m_{X} \cdot T a \cdot m_{X}^{\circ}(\mathfrak{x}, y) \\
& =\psi \cdot T a \cdot m_{X}^{\circ}(\mathfrak{x}, y)=\psi(\mathfrak{x}, y) .
\end{aligned}
$$

Now assume that $\psi:|X| \otimes Y \rightarrow \mathrm{V}$ and $\psi: X^{\mathrm{op}} \otimes Y \rightarrow \mathrm{V}$ are $(\mathbb{T}, \mathrm{V})$-functors. Functoriality of $\psi:|X| \otimes Y \rightarrow \vee$ implies, for all $\mathfrak{x} \in T X$ and $y \in Y$,

$$
\begin{aligned}
\psi(\mathfrak{x}, y) \geq & \bigvee_{\substack{\mathfrak{X} \in T T X: \\
m_{X}(\mathfrak{X})=\mathfrak{x} ; \\
\mathfrak{y} \in T Y}} \bigvee\left\{\xi \cdot T_{\mathrm{o}} \psi(\mathfrak{W}) \otimes b(\mathfrak{y}, y) \mid \mathfrak{W} \in T(T X \times Y): \mathfrak{W} \mapsto \mathfrak{X}, \mathfrak{W} \mapsto \mathfrak{y}\right\} \\
= & \bigvee_{\substack{\mathfrak{X} \in T T X: \\
m_{X}(\mathfrak{X})=\mathfrak{x} ; \\
\mathfrak{y} \in T Y}} T \psi(\mathfrak{X}, \mathfrak{y}) \otimes b(\mathfrak{y}, y) \\
= & \bigvee_{\substack{\mathfrak{X} \in T T X: \\
m_{X}(\mathfrak{X})=\mathfrak{x}}} b \cdot T \psi(\mathfrak{X}, y) \\
= & b \cdot T \psi \cdot m_{X}^{\circ}(\mathfrak{x}, y) .
\end{aligned}
$$

On the other hand, by functoriality of $\psi: X^{\mathrm{op}} \otimes Y \rightarrow \mathrm{\vee}$, for all $\mathfrak{x} \in T X$ and $y \in Y$ we have

$$
\begin{aligned}
\psi(\mathfrak{x}, y) & \geq \bigvee_{\substack{\mathfrak{X} \in T T X \\
\mathfrak{y} \in T Y}} \bigvee\left\{\xi \cdot T_{\mathrm{o}} \psi(\mathfrak{W}) \otimes b(\mathfrak{y}, y) \otimes a^{\mathrm{op}}(\mathfrak{X}, \mathfrak{x}) \mid \mathfrak{W} \in T(T X \times Y): \mathfrak{W} \mapsto \mathfrak{X}, \mathfrak{W} \mapsto \mathfrak{y}\right\} \\
& =\bigvee_{\substack{\mathfrak{X} \in T T X \\
\mathfrak{y} \in T Y}} T \psi(\mathfrak{X}, \mathfrak{y}) \otimes b(\mathfrak{y}, y) \otimes T^{2} a \cdot T m_{X}^{\circ} \cdot e_{T X}(\mathfrak{x}, \mathfrak{X}) \\
& =b \cdot T \psi \cdot T^{2} a \cdot T m_{X}^{\circ} \cdot e_{T X}(\mathfrak{x}, \mathfrak{X}) \\
& \geq b \cdot e_{Y} \cdot \psi \cdot T a \cdot m_{X}^{\circ}(\mathfrak{x}, y) \\
& \geq \psi \cdot T a \cdot m_{X}^{\circ}(\mathfrak{x}, y) .
\end{aligned}
$$

\section{4 $\mathrm{V}$ is Lawvere-complete.}

Theorem. Assume that $T 1=1$. Then $\left(V, \operatorname{hom}_{\xi}\right)$ is a Lawvere-complete $(\mathbb{T}, \mathrm{V})$-category provided that $a:=M^{\circ}\left(\operatorname{hom}_{\xi}\right)=\xi^{\circ} \cdot \operatorname{hom} \cdot \xi$ (i.e., for $\left.\mathfrak{v}, \mathfrak{w} \in T \vee, a(\mathfrak{v}, \mathfrak{w})=\operatorname{hom}(\xi(\mathfrak{v}), \xi(\mathfrak{w}))\right)$.

Proof. Let

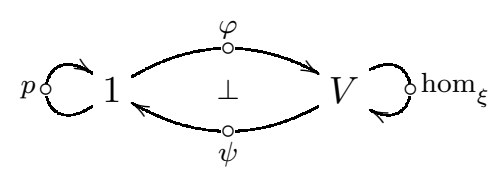

be a pair of adjoint bimodules. By the previous theorem we know that:

$$
\begin{aligned}
\varphi \text { bimodule } & \Longleftrightarrow \varphi:\left(\mathrm{V}, \operatorname{hom}_{\xi}\right) \rightarrow\left(\mathrm{V}, \operatorname{hom}_{\xi}\right) \text { is a }(\mathbb{T}, \mathrm{V}) \text {-functor } \\
& \Longleftrightarrow \forall \mathfrak{v} \in T \vee \forall v \in \mathrm{V} \operatorname{hom}(\xi(\mathfrak{v}), v) \leq \operatorname{hom}(\xi \cdot T \varphi(\mathfrak{v}), \varphi(v))
\end{aligned}
$$


In particular, for every $\mathfrak{v} \in T \bigvee, k \leq \operatorname{hom}(\xi(\mathfrak{v}), \xi(\mathfrak{v})) \leq \operatorname{hom}(\xi \cdot T \varphi(\mathfrak{v}), \varphi \cdot \xi(\mathfrak{v}))$, hence $\xi \cdot T \varphi(\mathfrak{v}) \leq$ $\varphi \cdot \xi(\mathfrak{v})$.

$$
\begin{aligned}
\psi \text { bimodule } & \Longleftrightarrow \psi:\left(T \vee, a^{\circ}\right) \rightarrow(V, \text { hom }) \text { is a } \vee \text {-functor } \\
& \Longleftrightarrow \forall \mathfrak{v}, \mathfrak{w} \in T \vee a(\mathfrak{v}, \mathfrak{w}) \leq \operatorname{hom}(\psi(\mathfrak{w}), \psi(\mathfrak{v})) .
\end{aligned}
$$

Finally,

(6) $\quad \dashv \psi \Longleftrightarrow\left\{\begin{array}{l}(a) \quad \varphi * \psi \leq \operatorname{hom} \cdot \xi \Longleftrightarrow \forall \mathfrak{v} \in T \vee \forall v \in \mathrm{V} \psi(\mathfrak{v}) \otimes \varphi(v) \leq \operatorname{hom}(\xi(\mathfrak{v}), v), \\ (b) \quad p \leq \psi * \varphi \Longleftrightarrow k \leq \bigvee_{\mathfrak{u} \in T V} \psi(\mathfrak{u}) \otimes \xi\left(T_{\mathrm{o}} \varphi(\mathfrak{u})\right) .\end{array}\right.$

We will show that the adjunction $\varphi \dashv \psi$ is represented by $\psi(\dot{k})$, where $\dot{k}=e_{\mathrm{V}}(k)$. Similarly to the proof of Theorem 1.6, we split our argument in three steps:

(1st) $\psi(\dot{k})=\bigvee_{\mathfrak{v} \in T V} \psi(\mathfrak{v}) \otimes \xi(\mathfrak{v})$ :

" $\leq$ " is immediate; for " $\geq$ " we argue as follows:

$$
\begin{aligned}
\psi(\mathfrak{v}) \otimes \xi(\mathfrak{v}) & =\psi(\mathfrak{v}) \otimes \operatorname{hom}(\xi(\dot{k}), \xi(\mathfrak{v})) \\
& =\psi(\mathfrak{v}) \otimes a(\dot{k}, \mathfrak{v}) \\
& \leq \psi(\mathfrak{v}) \otimes \operatorname{hom}(\psi(\mathfrak{v}), \psi(\dot{k})) \\
& \leq \psi(\dot{k}) .
\end{aligned}
$$

(2nd) $\forall \mathfrak{v} \in T V \operatorname{hom}_{\xi}(\mathfrak{v}, \psi(\dot{k}))=\bigvee_{\mathfrak{u} \in T V} \operatorname{hom}(\xi(\mathfrak{v}), \xi(\mathfrak{u})) \otimes \psi(\mathfrak{u})$ :

To check " $\geq$ " we just observe that

$$
\xi(\mathfrak{v}) \otimes(\operatorname{hom}(\xi(\mathfrak{v}), \xi(\mathfrak{u})) \otimes \psi(\mathfrak{u})) \leq \xi(\mathfrak{u}) \otimes \psi(\mathfrak{u}) \leq \psi(\dot{k}) .
$$

For " $\leq$ ", first note that

$$
\psi(\dot{k}) \otimes \varphi(\xi(\mathfrak{u})) \leq \operatorname{hom}(\xi(\dot{k}), \xi(\mathfrak{u}))=\operatorname{hom}(k, \xi(\mathfrak{u}))=\xi(\mathfrak{u})
$$

from which follows

$$
\xi(T \varphi(\mathfrak{u})) \leq \varphi(\xi(\mathfrak{u})) \leq \operatorname{hom}(\psi(\dot{k}), \xi(\mathfrak{u})) .
$$

From that we conclude that

$$
\begin{aligned}
& \operatorname{hom}(\xi(\mathfrak{v}), \psi(\dot{k})) \leq \operatorname{hom}(\xi(\mathfrak{v}), \psi(\dot{k})) \otimes \bigvee_{\mathfrak{u} \in T V} \psi(\mathfrak{u}) \otimes \xi(T \varphi(\mathfrak{u})) \\
& =\bigvee_{\mathfrak{u} \in T V} \operatorname{hom}(\xi(\mathfrak{v}), \psi(\dot{k})) \otimes \psi(\mathfrak{u}) \otimes \xi(T \varphi(\mathfrak{u})) \\
& \left.\leq \bigvee_{\mathfrak{u} \in T V} \operatorname{hom}(\xi(\mathfrak{v}), \psi(\dot{k})) \otimes \operatorname{hom}(\psi(\dot{k}), \xi(\mathfrak{u})) \otimes \psi(\mathfrak{u}) \quad \text { (by (7) }\right) \\
& \leq \bigvee_{\mathfrak{u} \in T V} \operatorname{hom}(\xi(\mathfrak{v}), \xi(\mathfrak{u})) \otimes \psi(\mathfrak{u})
\end{aligned}
$$


Lemma. Assume that $T 1=1$. Then $T\left(\operatorname{hom}_{\xi}\right) \cdot m_{\vee}^{\circ}=\xi^{\circ} \cdot \operatorname{hom} \cdot \xi$ provided that

$$
\xi \cdot T_{\mathrm{o}} \operatorname{hom}\left(u,_{-}\right) \leq \operatorname{hom}\left(u,_{-}\right) \cdot \xi \text {, }
$$

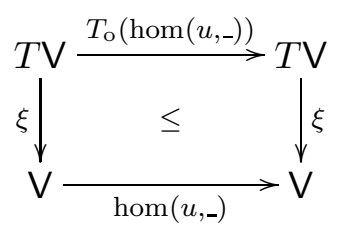

for each $u \in \mathrm{V}$. The inequality (8) is surely true if hom $(u,-)$ preserves non-empty suprema.

Proof. First observe that

$$
\begin{aligned}
T\left(\operatorname{hom}_{\xi}\right) \cdot m_{V}^{\circ} & =T \operatorname{hom} \cdot T \xi \cdot m_{\mathrm{V}}^{\circ} \\
& \leq \xi^{\circ} \cdot \operatorname{hom} \cdot \xi \cdot T \xi \cdot m_{\mathrm{V}}^{\circ} \quad \text { (because } \xi \text { is a } \mathrm{V} \text {-functor, by Proposition 3.2) } \\
& =\xi^{\circ} \cdot \operatorname{hom} \cdot \xi \cdot m_{\mathrm{V}} \cdot m_{V}^{\circ} \\
& \leq \xi^{\circ} \cdot \operatorname{hom} \cdot \xi .
\end{aligned}
$$

On the other hand, for $\mathfrak{u}, \mathfrak{v} \in T \vee$, we have

$$
\begin{aligned}
a(\mathfrak{u}, \mathfrak{v}) & \geq T \operatorname{hom}_{\xi}(\dot{\mathfrak{u}}, \mathfrak{v}) \\
& =T \operatorname{hom}\left(T_{\mathrm{o}} \xi(\dot{\mathfrak{u}}), \mathfrak{v}\right) \\
& =T \operatorname{hom}(\dot{\xi}(\dot{\mathfrak{u}}), \mathfrak{v}) \\
& =\xi \cdot T_{\mathrm{o}} \operatorname{hom} \cdot T_{\mathrm{o}}\langle\xi(\mathfrak{u}), 1 \mathfrak{v}\rangle(\mathfrak{v}) \\
& \geq \operatorname{hom}\left(\xi(\mathfrak{u}), \_\right) \cdot \xi(\mathfrak{v})=\operatorname{hom}(\xi(\mathfrak{u}), \xi(\mathfrak{v})) .
\end{aligned}
$$

To see $(*)$, just observe that $T_{\mathrm{o}}\left\langle\xi(\mathfrak{u}), 1_{\mathrm{V}}\right\rangle(\mathfrak{v})$ is the only element of $T(\mathrm{~V} \times \mathrm{V})$ which projects to both $\dot{\xi(\mathfrak{u})}$ and $\mathfrak{v}$. Assume now that $\operatorname{hom}\left(u,{ }_{-}\right)$preserves non-empty suprema and let $u \in \mathrm{V}$ and $\mathfrak{u} \in T \mathrm{~V}$. Then

$$
\begin{aligned}
\operatorname{hom}(u, \xi(\mathfrak{u})) & =\operatorname{hom}(u, \bigvee\{v \in \mathrm{V} \mid \mathfrak{u} \in T(\uparrow v)\}) \\
& =\bigvee\{\operatorname{hom}(u, v) \mid v \in \mathrm{V}, \mathfrak{u} \in T(\uparrow v)\}) \\
& \left.\leq \bigvee\left\{v \in \mathrm{V} \mid T_{\mathrm{o}} \operatorname{hom}\left(u,_{-}\right)(\mathfrak{u}) \in T(\uparrow v)\right\}\right)
\end{aligned}
$$

\section{A Yoneda Lemma for $(\mathbb{T}, V)$-categories}

4.1 Function spaces. In this section we wish to obtain the analogue result to Corollary 1.5 in the setting of $(\mathbb{T}, V)$-categories. This in turn requires an understanding of the right adjoint to $X \otimes_{-}:(\mathbb{T}, \mathrm{V})$-Cat $\rightarrow(\mathbb{T}, \mathrm{V})$-Cat, a problem studied in [16]. From there we import the following result.

Proposition. Let $X=(X, a)$ be a $(\mathbb{T}, \bigvee)$-category. Then $X \otimes_{-}:(\mathbb{T}, \bigvee)$-Cat $\rightarrow(\mathbb{T}, \mathrm{V})$-Cat has a right adjoint ${ }_{-} X$ provided that $a \cdot T a=a \cdot m_{X}$.

Certainly, each (Eilenberg-Moore) $\mathbb{T}$-algebra, considered as a $(\mathbb{T}, V)$-category, satisfies the condition above. Moreover, the $(\mathbb{T}, \mathrm{V})$-categorical structure $(X, a)$ induced by any $\mathrm{V}$-category $X=(X, r)$ (see 2.3) satisfies this condition if $T e_{X} \cdot e_{X}=m_{X}^{\circ} \cdot e_{X}$. 
Let $X=(X, a)$ and $(Y, b)$ be $(\mathbb{T}, \mathrm{V})$-categories, and assume that $a \cdot T a=a \cdot m_{X}$. Then $Y^{X}$ has as underlying set

$$
\{h:(X, a) \otimes(1, p) \rightarrow(Y, b) \mid h \text { is a }(\mathbb{T}, \mathrm{V}) \text {-functor }\}
$$

thanks to the bijection (with $P=(1, p)$ )

$$
\frac{X \otimes P \rightarrow Y}{P \rightarrow Y^{X}}
$$

The structure $\llbracket a, b \rrbracket$ on $Y^{X}$ is the largest structure making the evaluation map

$$
\text { ev }: X \otimes Y^{X} \rightarrow Y,(x, h) \mapsto h(x)
$$

a (T, $\mathbb{V})$-functor: for $\mathfrak{p} \in T\left(Y^{X}\right)$ and $h \in Y^{X}$ we have

$$
\llbracket a, b \rrbracket(\mathfrak{p}, h)=\bigvee\left\{v \in \mathrm{V} \mid \forall \mathfrak{q} \in T \pi_{Y^{X}}^{-1}(\mathfrak{p}), x \in X \quad a\left(T \pi_{X}(\mathfrak{q}), x\right) \otimes v \leq b(T \operatorname{ev}(\mathfrak{q}), h(x))\right\} .
$$

4.2 The Yoneda Embedding. By Theorem 3.3, the bimodule $a: X \rightarrow X$ gives rise to $(\mathbb{T}, \mathrm{V})$-functors $a:|X| \otimes X \rightarrow \mathrm{V}$ and $a: X^{\mathrm{op}} \otimes X \rightarrow \mathrm{V}$. According to the considerations above, we obtain a $(\mathbb{T}, \mathrm{V})$-functor $y=\ulcorner a\urcorner: X \rightarrow \mathrm{V}^{|X|}$. Our next result should be compared with Corollary 1.5 .

Theorem (Yoneda). Let $X=(X, a)$ be a $(\mathbb{T}, \mathrm{V})$-category. Then the following assertions hold.

(a) For all $\mathfrak{x} \in T X$ and $\varphi \in \mathrm{V}^{|X|}, \llbracket m_{X}, \operatorname{hom}_{\xi} \rrbracket(T y(\mathfrak{x}), \varphi) \leq \varphi(\mathfrak{x})$.

(b) Let $\varphi \in \mathrm{V}^{|X|}$. Then

$$
\forall \mathfrak{x} \in T X \varphi(\mathfrak{x}) \leq \llbracket m_{X}, \operatorname{hom}_{\xi} \rrbracket(T y(\mathfrak{x}), \varphi) \quad \Longleftrightarrow \quad \varphi: X^{\mathrm{op}} \rightarrow \mathrm{V} \text { is a }(\mathbb{T}, \mathrm{V}) \text {-functor }
$$

Proof. Note that the diagrams
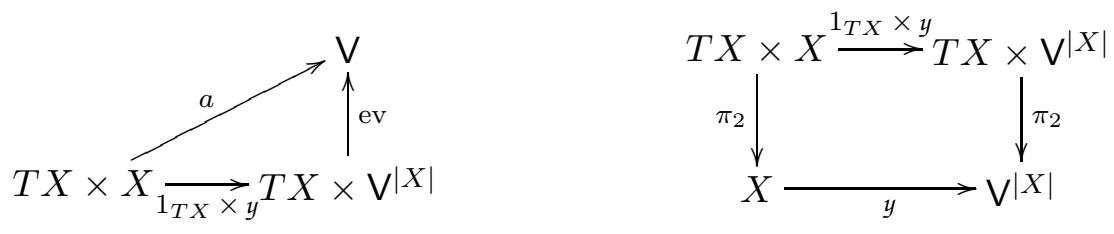

commute, where the right-hand side diagram is even a pullback. Let $\mathfrak{x} \in T X$ and $\varphi \in \mathrm{V}^{|X|}$. 
Hence

$$
\begin{aligned}
& \llbracket m_{X}, \operatorname{hom}_{\xi} \rrbracket\left(T_{\mathrm{o}} y(\mathfrak{x}), \varphi\right) \\
& =\bigvee\left\{v \in \mathrm{V} \mid \forall \mathfrak{y} \in T X, \mathfrak{Y} \in m_{X}^{-1}(\mathfrak{y}), \mathfrak{W} \in T\left(T X \times \bigvee^{|X|}\right)\right. \\
& \left.\left(T_{\mathrm{o}} \pi_{1}(\mathfrak{W})=\mathfrak{Y} \& T_{\mathrm{o}} \pi_{2}(\mathfrak{W})=T_{\mathrm{o}} y(\mathfrak{x})\right) \Rightarrow v \leq \operatorname{hom}\left(\xi \cdot T_{\mathrm{o}} \operatorname{ev}(\mathfrak{W}), \varphi(\mathfrak{y})\right)\right\} \\
& =\bigvee\left\{v \in \mathrm{V} \mid \forall \mathfrak{y} \in T X, \mathfrak{Y} \in m_{X}^{-1}(\mathfrak{y}), \mathfrak{W} \in T(T X \times X)\right. \\
& \left.\left(T_{\mathrm{o}} \pi_{1}(\mathfrak{W})=\mathfrak{Y} \& T_{\mathrm{o}} \pi_{2}(\mathfrak{W})=\mathfrak{x}\right) \Rightarrow v \leq \operatorname{hom}\left(\xi \cdot T_{\mathrm{o}} a(\mathfrak{W}), \varphi(\mathfrak{y})\right)\right\} \\
& =\bigvee\left\{v \in \mathrm{V} \mid \forall \mathfrak{y} \in T X, \mathfrak{Y} \in m_{X}^{-1}(\mathfrak{y}) \quad v \leq \bigwedge_{\substack{\mathfrak{W} \in T(T X \times X) \\
T_{\mathrm{o}} \pi_{1}(\mathfrak{W})=\mathfrak{Y} \\
T_{\mathrm{o}} \pi_{2}(\mathfrak{W})=\mathfrak{x}}} \operatorname{hom}\left(\xi \cdot T_{\mathrm{o}} a(\mathfrak{W}), \varphi(\mathfrak{y})\right)\right\} \\
& =\bigvee\left\{v \in \mathrm{V} \mid \forall \mathfrak{y} \in T X, \mathfrak{Y} \in m_{X}^{-1}(\mathfrak{y}) \quad v \leq \operatorname{hom}\left(\bigvee_{\substack{\mathfrak{W} \in T(T X \times X) \\
T_{\mathrm{o}} \pi_{1}(\mathfrak{W})=\mathfrak{Y} \\
T_{\mathrm{o}} \pi_{2}(\mathfrak{W})=\mathfrak{x}}} \xi \cdot T_{\mathrm{o}} a(\mathfrak{W}), \varphi(\mathfrak{y})\right)\right\} \\
& =\bigvee\left\{v \in \mathrm{V} \mid \forall \mathfrak{y} \in T X, \mathfrak{Y} \in m_{X}^{-1}(\mathfrak{y}) \quad v \leq \operatorname{hom}(T a(\mathfrak{Y}, \mathfrak{x}), \varphi(\mathfrak{y}))\right\} \\
& =\bigvee\left\{v \in \mathrm{V} \mid \forall \mathfrak{y} \in T X \quad T a \cdot m_{X}^{\circ}(\mathfrak{y}, \mathfrak{x}) \otimes v \leq \varphi(\mathfrak{y})\right\} .
\end{aligned}
$$

In particular we have

$$
v=k \otimes v \leq T a \cdot m_{X}^{\circ}(\mathfrak{x}, \mathfrak{x}) \otimes v \leq \varphi(\mathfrak{x}),
$$

which proves (a). On the other hand, $\varphi:\left(T X, T a \cdot m_{X}^{\circ}\right) \rightarrow(\mathrm{V}$, hom $)$ is a $\mathrm{V}$-functor if and only if

$$
T a \cdot m_{X}^{\circ}(\mathfrak{y}, \mathfrak{x}) \otimes \varphi(\mathfrak{x}) \leq \varphi(\mathfrak{y})
$$

for all $\mathfrak{y}, \mathfrak{x} \in T X$, from which follows (b).

We put $\hat{X}=(\hat{X}, \hat{a})$ where $\hat{X}:=\left\{\varphi \in \mathrm{V}^{|X|} \mid \varphi: X^{\mathrm{op}} \rightarrow \mathrm{V}\right.$ is a $(\mathbb{T}, \mathrm{V})$-functor $\}$ considered as a subcategory of $\mathrm{V}^{|X|}$. Recall that $a: X^{\mathrm{op}} \otimes X \rightarrow \mathrm{V}$ is a $(\mathbb{T}, \mathrm{V})$-functor, and therefore $a(-, x): X^{\mathrm{op}} \otimes P \rightarrow \mathrm{V}$ is a $(\mathbb{T}, \mathrm{V})$-functor for each $x \in X$. If $T 1=1$, then $P=(1, p)=(1, k)$ is the neutral element for $\otimes$ and we can restrict the Yoneda functor $y$ to $\hat{X}$.

Corollary. Assume $T 1=1$. Then the Yoneda functor $y: X \rightarrow \hat{X}$ is full and faithful.

If $T e_{X} \cdot e_{X}=m_{X}^{\circ} \cdot e_{X}$, we might also consider the transpose $y_{0}=\ulcorner a\urcorner: X \rightarrow \mathrm{V}^{X^{\mathrm{op}}}$ of $a: X^{\mathrm{op}} \otimes X \rightarrow \mathrm{V}$ as below. However, unlike the situation for $\mathrm{V}$-categories, in general we do not have $\hat{X} \cong \mathrm{V}^{X^{\mathrm{op}}}$ (see example below).

Proposition (Yoneda II). Assume that $T e_{X} \cdot e_{X}=m_{X}^{\circ} \cdot e_{X}$ and let $X=(X, a)$ be $a(\mathbb{T}, \mathrm{V})$ category. Then the following assertions hold.

(a) For all $\mathfrak{x} \in T X$ and $\varphi \in \mathrm{V}^{X^{\mathrm{op}}}, \llbracket a^{\mathrm{op}}, \operatorname{hom}_{\xi} \rrbracket\left(T y_{0}(\mathfrak{x}), \varphi\right) \geq \varphi(\mathfrak{x})$.

(b) Let $\mathfrak{x} \in T X$ such that $T a \cdot e_{T X}(\mathfrak{x}, \mathfrak{x}) \geq k$. Then $\llbracket a^{\mathrm{op}}, \operatorname{hom}_{\xi} \rrbracket\left(T y_{0}(\mathfrak{x}), \varphi\right) \leq \varphi(\mathfrak{x})$. 
Proof. Let $\mathfrak{x} \in T X$ and $\varphi \in \mathrm{V}^{X^{\text {op }}}$. As above, we obtain

$$
\begin{aligned}
& \llbracket a^{\mathrm{op}}, \operatorname{hom}_{\xi} \rrbracket\left(T_{\mathrm{o}} y(\mathfrak{x}), \varphi\right) \\
& =\bigvee\left\{v \in \mathrm{V} \mid \forall \mathfrak{y} \in T X, \mathfrak{Y} \in T^{2} X, \mathfrak{W} \in T\left(T X \times \mathrm{V}^{X^{\mathrm{op}}}\right)\right. \\
& \left.\quad\left(T_{\mathrm{o}} \pi_{1}(\mathfrak{W})=\mathfrak{Y} \& T_{\mathrm{o}} \pi_{2}(\mathfrak{W})=T_{\mathrm{o}} y(\mathfrak{x})\right) \Rightarrow a^{\mathrm{op}}(\mathfrak{Y}, \mathfrak{x}) \otimes v \leq \operatorname{hom}\left(\xi \cdot T_{\mathrm{o}} \operatorname{ev}(\mathfrak{W}), \varphi(\mathfrak{y})\right)\right\} \\
& =\bigvee\left\{v \in \mathrm{V} \mid \forall \mathfrak{y} \in T X, \mathfrak{Y} \in T^{2} Y \cdot T a(\mathfrak{Y}, \mathfrak{x}) \otimes a^{\mathrm{op}}(\mathfrak{Y}, \mathfrak{y}) \otimes v \leq \varphi(\mathfrak{y})\right\} \\
& =\bigvee\left\{v \in \mathrm{V} \mid \forall \mathfrak{y} \in T X \quad a^{\mathrm{op}} \cdot T a^{\circ}(\mathfrak{x}, \mathfrak{y}) \otimes v \leq \varphi(\mathfrak{y})\right\} .
\end{aligned}
$$

Furthermore, we have

$$
a^{\mathrm{op}} \cdot T a^{\circ}=e_{T X}^{\circ} \cdot T m_{X}^{\circ} \cdot T T a^{\circ} \cdot T a^{\circ}=e_{T X}^{\circ} \cdot T a^{\circ} \leq m_{X} \cdot T a^{\circ} .
$$

Hence $\varphi(\mathfrak{x}) \leq \llbracket a^{\mathrm{op}}, \operatorname{hom}_{\xi} \rrbracket\left(T_{\mathrm{o}} y_{0}(\mathfrak{x}), \varphi\right)$ and, if $k \leq T a \cdot e_{T X}(\mathfrak{x}, \mathfrak{x})=a^{\mathrm{op}} \cdot T a^{\circ}(\mathfrak{x}, \mathfrak{x})$, we also have $\llbracket a^{\mathrm{op}}, \operatorname{hom}_{\xi} \rrbracket\left(T_{\mathrm{o}} y_{0}(\mathfrak{x}), \varphi\right) \leq \varphi(\mathfrak{x})$.

Example. Unlike $y$, the functor $y_{0}$ does not need to be full and faithful. In fact, consider $X=\mathbb{N}$ as a $(\mathbb{U}, 2)$-category, i.e. a topological space, equipped with the discrete topology $a=e_{\mathbb{N}}^{\circ}$. Then $\mathbb{N}^{\text {op }}$ is the discrete space $\mathbb{N}^{\text {op }}=\left(U \mathbb{N}, e_{U \mathbb{N}}^{o}\right)$. Let $\mathfrak{x}$ be a free ultrafilter on $\mathbb{N}$. Then, for each $\mathfrak{y} \in U \mathbb{N}, a^{\text {op }} \cdot U a^{\circ}(\mathfrak{x}, \mathfrak{y})=e_{\mathbb{N}}^{\circ} \cdot U e_{\mathbb{N}}(\mathfrak{x}, \mathfrak{y})=$ false and therefore $U y_{0}(\mathfrak{x}) \rightarrow \varphi$ for each $\varphi \in 2^{\mathbb{N}^{\text {op }}}$. On the other hand, for $\varphi=a(-, x)$ ( $x$ any element of $\mathbb{N})$ we have $\varphi(\mathfrak{x})=$ false. In particular we see that $y_{0}: \mathbb{N} \rightarrow 2^{\mathbb{N}^{o p}}$ is not full and faithful.

\section{Examples}

5.1 Ordered sets. Recall that 2-Cat $=$ Ord. Given an ordered set $X=(X, \leq)$, by Theorem 1.5 we have that a bimodule $\phi: 1 \multimap X$ is an order-preserving map $\phi: X \rightarrow 2$, while a bimodule $\psi: X \multimap \rightarrow 1$ is an order-preserving map $X^{\mathrm{op}} \rightarrow 2$. We can identify $\varphi$ with the upclosed set $A=\varphi^{-1}$ (true) and $\psi$ with the downclosed set $B=\psi^{-1}$ (true). Under this identification, $\varphi \dashv \psi$ translates to

$$
A \cap B \neq \varnothing \quad \text { and } \quad \forall x \in A \quad \forall y \in B \quad y \leq x .
$$

Then any $z \in A \cap B$ is simultaneously a smallest element of $A$ and a largest element of $B$, therefore $z$ represents $\varphi \dashv \psi$. Hence, by Proposition 1.6, each ordered set is Lawvere-complete. Note that the proof of Proposition 1.6 makes use of the Axiom of Choice; in fact, as pointed out in [6], here we have no choice.

Theorem. The following assertions are equivalent.

(i) Each ordered set is Lawvere-complete.

(ii) The Axiom of Choice.

Proof. To see (ii) $\Rightarrow$ (i), let $f: X \rightarrow Y$ be a surjective map. We equip $Y$ with the discrete order $\Delta_{Y}$ and $X$ with the kernel relation of $f$; then we have not only $f_{*} \dashv f^{*}$ but also $f^{*} \dashv f_{*}$. Hence there exists some $g: Y \rightarrow X$ which represents $f^{*} \dashv f_{*}$, and such $g$ necessarily satisfies $f \cdot g=1_{Y}$. 
5.2 Metric spaces. For $\mathrm{V}=\mathrm{P}_{+}$we have $\mathrm{P}_{+}$-Cat $\cong$ Met. Let $X=(X, d)$ be a metric space. A pair of adjoint bimodules $\varphi \dashv \psi$ corresponds to a pair of non-expansive maps $\varphi: X \rightarrow \mathrm{P}_{+}$and $\psi: X^{\mathrm{op}} \rightarrow \mathrm{P}_{+}$which satisfy

$$
\inf _{x \in X} \varphi(x)+\psi(x)=0 \quad \text { and } \quad \forall x, y \in X \quad \psi(y)+\varphi(x) \geq d(y, x) .
$$

As observed in [18], pairs of adjoint bimodules on $X$ correspond exactly to equivalence classes of Cauchy sequences. To see this, recall first that a sequence $s=\left(x_{n}\right)_{n \in \mathbb{N}}$ is called Cauchy if

$$
\inf _{k \in \mathbb{N}} \sup _{n, n^{\prime} \geq k} d\left(x_{n}, x_{n^{\prime}}\right)=0 .
$$

Given a Cauchy sequence $s=\left(x_{n}\right)_{n \in \mathbb{N}}$, we have

$$
\inf _{m \in \mathbb{N}} \sup _{n \geq m} d\left(x_{n}, x\right)=\sup _{m \in \mathbb{N}} \inf _{n \geq m} d\left(x_{n}, x\right)
$$

as well as

$$
\inf _{m \in \mathbb{N}} \sup _{n \geq m} d\left(x, x_{n}\right)=\sup _{m \in \mathbb{N}} \inf _{n \geq m} d\left(x, x_{n}\right),
$$

and $s$ gives rise to non-expansive maps

$$
\begin{aligned}
& \varphi_{s}: X \rightarrow \mathrm{P}_{+} \\
& x \mapsto \sup _{m \in \mathbb{N}} \inf _{n \geq m} d\left(x_{n}, x\right) \\
& \psi_{s}: X^{\mathrm{op}} \rightarrow \mathrm{P}_{+} \text {. } \\
& x \mapsto \sup _{m \in \mathbb{N}} \inf _{n \geq m} d\left(x, x_{n}\right)
\end{aligned}
$$

One sees easily that $\varphi_{s} \dashv \psi_{s}$; moreover, two equivalent Cauchy sequences induce the same maps.

On the other hand, given an adjunction $\varphi \dashv \psi$, we may define $s=\left(x_{n}\right)_{n \in \mathbb{N}}$ such that $\varphi\left(x_{n}\right)+\psi\left(x_{n}\right) \leq \frac{1}{n}$, hence $d\left(x_{n}, x_{m}\right) \leq \frac{1}{n}+\frac{1}{m}$, and therefore $s$ is a Cauchy sequence. Any two such sequences are equivalent. Furthermore, $\varphi \leq \varphi_{s}$ as well as $\psi \leq \psi_{s}$, therefore, since $\varphi \dashv \psi$ and $\varphi_{s} \dashv \psi_{s}$, we have even equality. Starting with a Cauchy sequence $s=\left(x_{n}\right)_{n \in \mathbb{N}}$, then for any sequence $t=\left(y_{n}\right)_{n \in \mathbb{N}}$ chosen for $\varphi \dashv \psi$ as above we have

$$
\inf _{m \in \mathbb{N}} \inf _{k \in \mathbb{N}} \sup _{n \geq k} d\left(x_{n}, y_{m}\right)=0 \quad \text { and } \quad \inf _{m \in \mathbb{N}} \inf _{k \in \mathbb{N}} \sup _{n \geq k} d\left(y_{m}, x_{n}\right)=0,
$$

hence $s$ and $t$ are equivalent. Finally, $s=\left(x_{n}\right)_{n \in \mathbb{N}}$ converges to $x$ (i.e. $s$ is equivalent to $(x)_{n \in \mathbb{N}}$ ) if and only if $\varphi_{s} \dashv \psi_{s}$ is represented by $x$.

The same argumentation applies also to the case $\mathrm{V}=\mathrm{P}_{\max }$ : pairs of adjoint bimodules $\varphi \dashv \psi: 1 \rightarrow X$ with $X$ an ultrametric space correspond precisely to Cauchy sequences in $X$, and convergence to representability.

Remark. A notion of non-symmetric Cauchy-sequence was introduced and studied in [3].

5.3 Topological spaces. We consider now $\mathbb{T}=\mathbb{U}=(U, e, m)$ the ultrafilter monad and $\mathrm{V}=2$. As already stated, Proposition 3.1 describes our extension $U$ in terms of $U_{\mathrm{o}}:$ Set $\rightarrow$ Set (for a direct calculation of $U$, see [7, Example 6.4]). Then (UU,2)-Cat $=$ Top, as it was shown by Barr [1]. By Theorem 3.3, a bimodule $\varphi: U 1 \rightarrow X$ from the one-element space 1 to a topological space $X$ is essentially a continuous map $\varphi: X \rightarrow 2$ from $X$ into the Sierpinski space 2, hence we can identify it with a closed subset $A \subseteq X$. A bimodule $\psi: U X \rightarrow 1$ is basically a map $\psi: U X \rightarrow 2$ such that $\mathcal{A}=\psi^{-1}$ (true) is closed in $|X|$ as well as in $X^{\mathrm{op}}$. The topology on $|X|$ 
is given by the Zariski closure, that is, $\mathfrak{x} \in U X$ is in the closure of $\mathcal{M} \subseteq U X$ if $\bigcap \mathcal{M} \subseteq \mathfrak{x}$. To understand the structure of $X^{\mathrm{op}}$, observe first that the order on $M^{\circ} X$ is given by

$$
\begin{aligned}
\mathfrak{x} \leq \mathfrak{y} & \Longleftrightarrow \exists \mathfrak{X} \in U^{2} X m_{X}(\mathfrak{X})=\mathfrak{x} \text { and } \mathfrak{X} \rightarrow \mathfrak{y} \\
& \Longleftrightarrow \forall A \in \mathfrak{x}, B \in \mathfrak{y} \quad \exists \mathfrak{a} \in U A, y \in B \mathfrak{a} \rightarrow y \\
& \Longleftrightarrow \forall A \in \mathfrak{x}, B \in \mathfrak{y} \quad \bar{A} \cap B \neq \varnothing .
\end{aligned}
$$

Denoting the filter base $\{\bar{A} \mid A \in \mathfrak{x}\}$ by $\overline{\mathfrak{x}}$, we have

$$
\mathfrak{x} \leq \mathfrak{y} \Longleftrightarrow \overline{\mathfrak{x}} \subseteq \mathfrak{y}
$$

Hence bimodules $\psi: U X \multimap 1$ can be identified with subsets $\mathcal{A} \subseteq U X$ which are Zariski closed and down-closed for the order described above. Now $\varphi \dashv \psi$ translates to

$$
\exists \mathfrak{x}_{0} \in U X \mathfrak{x}_{0} \in \mathcal{A} \& A \in \mathfrak{x}_{0} \quad \text { and } \quad \forall \mathfrak{x} \in \mathcal{A}, x \in A \mathfrak{x} \rightarrow x .
$$

Clearly, each $\mathfrak{x} \in \mathcal{A}$ converges to all points of $A$. On the other hand, for any $\mathfrak{x} \in U X$ with this property we have $\mathfrak{x} \leq \mathfrak{x}_{0}$ and therefore $\mathfrak{x} \in \mathcal{A}$. We conclude that

$$
\mathcal{A}=\{\mathfrak{x} \in U X \mid \forall x \in A \mathfrak{x} \rightarrow x\}
$$

A closed subset $A \subseteq X$ admits an ultrafilter $\mathfrak{x}_{0} \in U A$ which converges to all $x \in A$ if and only if $\{V \subseteq X \mid V$ open, $V \cap A \neq \varnothing\}$ is a filter base. In the language of closed sets this is expressed by saying that $A$ is not the union of two proper closed subsets, i.e. $A$ is irreducible. Finally, $\psi$ (and hence $\varphi$ ) is representable if and only if $\mathfrak{x}_{0}$ can be chosen principal, that is, if and only if there exists some point $x_{0} \in A$ which converges to all $x \in A$. In conclusion, we have

Theorem. The following assertions are equivalent for a topological space $X$.

(i) $X$ is Lawvere-complete.

(ii) Each irreducible closed subset $A \subseteq X$ is of the form $A=\overline{\{x\}}$ for some $x \in A$, i.e. $X$ is weakly sober.

5.4 Approach spaces. Recall that $A p p=\left(\mathbb{U}, P_{+}\right)$-Cat is the category of approach spaces and non-expansive maps. We fix an approach space $X=(X, a)$. As above, a bimodule $\varphi: U 1 \multimap X$ is a non-expansive map $\varphi: X \rightarrow \mathrm{P}_{+}$, by Theorem 3.3. There is a bijective correspondence between maps $\varphi: X \rightarrow \mathrm{P}_{+}$and families $\left(A_{v}\right)_{v \in \mathrm{P}_{+}}$of subsets $A_{v} \subseteq X$ satisfying

$$
A_{v}=\bigcap_{u>v} A_{u}
$$

where $\varphi \mapsto\left(\varphi^{-1}([0, v])\right)_{v \in \mathrm{P}_{+}}$and a family $\left(A_{v}\right)_{v \in \mathrm{P}_{+}}$defines the map $x \mapsto \inf \left\{v \in \mathrm{P}_{+} \mid x \in A_{v}\right\}$. Under this bijection, non-expansive maps correspond precisely to those families $\left(A_{v}\right)_{v \in \mathrm{P}_{+}}$which satisfy in addition

$$
\forall u, v \in \mathrm{P}_{+} \forall x \in X\left(d\left(A_{u}, x\right) \leq v \Rightarrow x \in A_{u+v}\right)
$$

where $d(A, x)=\inf \{a(\mathfrak{x}, x) \mid \mathfrak{x} \in U A\}$. 
We may think of the family $A=\left(A_{v}\right)_{v \in \mathrm{P}_{+}}$satisfying (9) as a variable set6; we call $A$ closed if it satisfies (10). Now it is not difficult to see that a right adjoint $\psi: X \nrightarrow \rightarrow 1$ to $\varphi: 1 \multimap X$ is determined by the variable set $\mathcal{A}=\left(\mathcal{A}_{v}\right)_{v \in \mathrm{P}_{+}}$given by

$$
\mathcal{A}_{v}=\left\{\mathfrak{x} \in U X \mid \forall u \in \mathrm{P}_{+} \quad \forall x \in A_{u} \quad a(\mathfrak{x}, x) \leq u+v\right\}
$$

for each $v \in \mathrm{P}_{+}$. Furthermore, given $\varphi: 1 \multimap X$, the variable set $\mathcal{A}$ defined as above corresponds to a right adjoint of $\varphi$ if and only if

$$
\forall u \in \mathrm{P}_{+}\left(u>0 \Rightarrow U A_{u} \cap \mathcal{A}_{u} \neq \varnothing\right)
$$

In analogy to the situation in Top, we call a variable set $A$ irreducible if it satisfies (11). Finally, we remark that the bimodule $\varphi: 1 \multimap X$ is represented by $x \in X$ precisely if the corresponding variable set $A$ is of the form

$$
A_{v}=\{y \in X \mid d(x, y) \leq v\},
$$

for each $v \in \mathrm{P}_{+}$. Naturally, we say that such a variable set is representable (by $x$ ).

Theorem. The following assertions are equivalent for an approach space $X$.

(i) $X$ is Lawvere-complete.

(ii) Each irreducible closed variable set $A$ is representable.

We point out that this setting satisfies the conditions of Theorem 3.4, therefore it assures that $\mathrm{P}_{+}$is Lawvere-complete.

Remark. The notion of approach frame and its connection with approach spaces was recently studied by Christophe Van Olmen in his $\mathrm{PhD}$ thesis [23]. In particular, the concept of sober approach space as a fixed point of the dual adjunction between App and the category AFrm of approach frames and homomorphisms was introduced. As confirmed by the author of [23], these are precisely the approach spaces where each irreducible closed variable set is uniquely representable.

\section{Appendix: Lawvere-complete quasi-uniform spaces}

6.1 Cauchy-complete quasi-uniform spaces. We recall that a quasi-uniformity $U$ on a set $X$ is a set of binary relations on $X$ such that:

$$
\begin{aligned}
& \forall u \in U \quad \Delta \subseteq u ; \\
& \forall u \in U \quad \exists v \in U \quad v \cdot v \subseteq u .
\end{aligned}
$$

The pair $(X, U)$ is called a quasi-uniform space; it is a uniform space when, for all $u \in U$, $u^{-1} \in U$. Given quasi-uniform spaces $(X, U)$ and $(Y, V)$, a map $f: X \rightarrow Y$ is uniformly continuous if

$$
\forall v \in V \quad \exists u \in U \quad \forall x, y \in X \quad x u y \Rightarrow f(x) v f(y) .
$$

\footnotetext{
${ }^{6}$ In fact, we may consider $A: \mathrm{P}_{+} \rightarrow$ Set as a sheaf where, for each $u \in \mathrm{P}_{+},\{v<u\}$ is a cover of $u$.
} 
Definition. Let $(X, U)$ be a quasi-uniform space.

1. A pair $(\mathfrak{f}, \mathfrak{g})$ is a filter in $(X, U)$ if $\mathfrak{f}$ and $\mathfrak{g}$ are filters in $X$ such that

$$
\forall F \in \mathfrak{f} \forall G \in \mathfrak{g} F \cap G \neq \varnothing .
$$

2. A filter $(\mathfrak{f}, \mathfrak{g})$ in $(X, U)$ is a Cauchy filter if

$$
\forall u \in U \quad \exists F \in \mathfrak{f} \exists G \in \mathfrak{g} F \times G \subseteq X_{u}:=\left\{\left(x, x^{\prime}\right) \mid x u x^{\prime}\right\} .
$$

3. A filter $(\mathfrak{f}, \mathfrak{g})$ in $(X, U)$ converges to $x_{0} \in X$ if

$$
\forall u \in U \quad \exists F \in \mathfrak{f} \exists G \in \mathfrak{g} F \times G \subseteq X_{-u x_{0}} \times X_{x_{0} u-},
$$

where $X_{-u x_{0}}:=\left\{x \in X \mid x u x_{0}\right\}$ and $X_{x_{0} u-}:=\left\{x \in X \mid x_{0} u x\right\}$.

Lemma. Given a quasi-uniformity $U$ in $X$ and $x_{0} \in X$, the neighbourhood filter of $x_{0}$

$$
\left(\left\{X_{-u x_{0}} \mid u \in U\right\},\left\{X_{x_{0} u-} \mid u \in U\right\}\right)
$$

is a minimal Cauchy filter in $(X, U)$.

Proposition. For a quasi-uniform space $(X, U)$, the following conditions are equivalent.

(i) Every Cauchy filter converges.

(ii) Every minimal Cauchy filter is the neighbourhood filter of a point $x_{0}$.

A quasi-uniform space is said to be Cauchy-complete if it satisfies any of the equivalent conditions of the Proposition.

For further information see [12] and [13].

6.2 Quasi-uniform spaces as lax algebras. In order to describe quasi-uniform spaces as lax algebras, we turn back to the setting described in [7] and substitute the bicategory V-Mat of 2.1 by the bicategory $Y$ having sets as objects and (possibly improper) filters in $\operatorname{Rel}(X, Y)$ as morphisms, where Rel is the bicategory of relations. The composition of two filters $R: X \longrightarrow Y$ and $S: Y \mapsto Z$ is the filter obtained by pointwise composition of relations $R \cdot S=\{s \cdot r \mid s \in S$ and $r \in R$ \}, while $R \leq R^{\prime}$ whenever $R^{\prime} \subseteq R$ (as sets).

We define a lax algebra now exactly like a $\mathrm{V}$-category: it is a $\mathrm{Y}$-morphism $A: X \rightarrow X$ such that

$$
1_{X} \leq A \text { and } A \cdot A \leq A
$$

or, equivalently,

$$
\forall x \in X \quad \forall a \in A \quad x a x \text { and } \forall a \in A \exists a^{\prime} \in A \quad a^{\prime} \cdot a^{\prime} \leq a .
$$

A lax morphism $f:(X, A) \rightarrow(Y, B)$ between lax algebras is a map $f: X \rightarrow Y$ such that $f \cdot A \leq B \cdot f$, i.e.

$$
\forall b \in B \quad \exists a \in A \quad f \cdot a \leq b \cdot f .
$$

It was shown in [7, Theorem 3.6] that this category of lax algebras and lax morphisms is equivalent to the category of quasi-uniform spaces and uniformly continuous maps. 
6.3 Adjoint pairs of bimodules in quasi-uniform spaces. A bimodule $\Psi:(X, A) \rightarrow(Y, B)$ between lax algebras is a $Y$-morphism $\Psi: X \mapsto Y$ such that $\Psi \cdot A \leq \Psi$ and $B \cdot \Psi \leq \Psi$. As in the context of $\mathrm{V}$-categories, $A$ and $B$ act as identities for the composition with bimodules, so that a pair of bimodules $(\Phi:(Y, B) \rightarrow(X, A),(\Psi:(X, A) \rightarrow(Y, B))$ is an adjoint pair, with $\Phi \dashv \Psi$, if $B \leq \Psi \cdot \Phi$ and $\Phi \cdot \Psi \leq A$. As before, every lax morphism $f:(X, A) \rightarrow(Y, B)$ defines a pair of adjoint bimodules $\left(f_{*}=B \cdot f:(X, A) \multimap(Y, B), f^{*}=f^{\circ} \cdot A:(Y, B) \multimap(X, A)\right)$. It is easy to check that Proposition 2.7 is still valid in this context.

Proposition. For a lax algebra $(X, A)$, the following conditions are equivalent.

(i) Each pair of adjoint bimodules $(\Phi:(Y, B) \multimap(X, A)) \dashv(\Psi:(X, A) \multimap(Y, B))$ is induced by a lax morphism $(Y, B) \rightarrow(X, A)$.

(ii) Each pair of adjoint bimodules $(\Phi: 1 \multimap(X, A)) \dashv(\Psi:(X, A) \multimap \rightarrow 1)$ is induced by a lax morphism $1 \rightarrow(X, A)$ (or simply a map).

Theorem. For Y-morphisms $\Phi: 1 \longrightarrow X$ and $\Psi: X \mapsto 1$, the following conditions are equivalent.

(i) $\Phi \dashv \Psi$.

(ii) $\left(\left\{X_{-\psi \star} \mid \psi \in \Psi\right\},\left\{X_{\star \varphi-} \mid \varphi \in \Phi\right\}\right)$ is a minimal Cauchy filter in $(X, A)$.

Proof. The conditions $1 \leq \Psi \cdot \Phi$ and $\Phi \cdot \Psi \leq A$ read as

$$
\begin{gathered}
\forall \psi \in \Psi \quad \exists \varphi \in \Phi \quad X_{\star \varphi-} \cap X_{-\psi \star} \neq \varnothing, \\
\forall a \in A \quad \exists \varphi \in \Phi \quad \exists \psi \in \Psi \quad X_{-\psi \star} \times X_{\star \varphi-} \subseteq X_{a},
\end{gathered}
$$

where the former condition means that $\left(\left\{X_{-{ }_{-}} \mid \psi \in \Psi\right\},\left\{X_{\star \varphi-} \mid \varphi \in \Phi\right\}\right)$ is a filter, while the latter one means that it is Cauchy.

(i) $\Rightarrow$ (ii): It remains to be shown that this Cauchy filter is minimal. Let $(\mathfrak{f}, \mathfrak{g})$ be a filter contained in it. If $\mathfrak{f} \varsubsetneqq\left\{X_{-\psi \star} \mid \psi \in \Psi\right\}$, i.e. if there exists $\psi \in \Psi$ such that $X_{-\psi \star} \notin \mathfrak{f}$, then there exist $a \in A$ and $\psi^{\prime} \in \Psi$ with $\psi^{\prime} \cdot a=\psi$, because $\psi$ is a bimodule, hence $a$ and $\psi^{\prime}$ are such that $\bigcup_{x^{\prime} \in X_{-\psi^{\prime} \star}} X_{-a x^{\prime}} \notin \mathfrak{f}$. Therefore

$$
\forall F \in \mathfrak{f} \exists x \in F \quad \forall x^{\prime} \in X_{-\psi^{\prime} \star}\left(x, x^{\prime}\right) \notin X_{a}
$$

Moreover, since

$$
\forall G \in \mathfrak{g} G \in\left\{X_{\star \varphi-} \mid \varphi \in \Phi\right\} \Rightarrow \forall G \in \mathfrak{g} \exists y \in X_{-\psi^{\prime} \star} \cap G,
$$

we obtain

$$
\forall F \in \mathfrak{f} \forall G \in \mathfrak{g} \exists x \in F \quad \exists y \in G(x, y) \notin X_{a},
$$

that is $(\mathfrak{f}, \mathfrak{g})$ is not a Cauchy filter.

(ii) $\Rightarrow$ (i): Let $\Phi: 1 \multimap(X, A)$ and $\Psi:(X, A) \multimap 1$ be a pair of bimodules and consider $\left(\left\{X_{-\psi \star} \mid \psi \in \Psi\right\},\left\{X_{\star \varphi-} \mid \varphi \in \Phi\right\}\right)$. We concluded already that the adjunction conditions are 
equivalent to this pair being a Cauchy filter. But we did not show yet that $\Phi$ and $\Psi$ are bimodules. For any $a \in A$,

$$
\left(\left\{\bigcup_{x \in X_{-\psi \star}} X_{-a x} \mid \psi \in \Psi, a \in A\right\},\left\{\bigcup_{y \in X_{\star \varphi-}} X_{y a-} \mid \varphi \in \Phi, a \in A\right\}\right)
$$

is a Cauchy filter contained in the former one, as we show next. First,

$$
\bigcup_{x \in X_{-\psi \star}} X_{-a x} \cap \bigcup_{y \in X_{\star a-}} X_{y a-} \supseteq X_{-\psi \star} \cap X_{\star \varphi-} \neq \varnothing .
$$

To prove the other condition, let $a \in A$, and consider $b \in A$ such that $b \cdot b \cdot b \leq a$. There exist $\varphi \in \Phi$ and $\psi \in \Psi$ such that $X_{-\psi \star} \times X_{\star \varphi-} \subseteq X_{b}$, and this implies that

$$
\bigcup_{x \in X_{-\psi \star}} X_{-b x} \times \bigcup_{y \in X_{\star \varphi-}} X_{y b-} \subseteq X_{a}
$$

since

$$
\begin{aligned}
& x^{\prime} \in \bigcup_{x \in X_{-\psi \star}} X_{-b x} \Rightarrow \exists x \in X_{-\psi \star}\left(x^{\prime}, x\right) \in X_{b}, \\
& y^{\prime} \in \bigcup_{y \in X_{\star \varphi-}} X_{y b-} \Rightarrow \exists y \in X_{\star \varphi-}\left(y, y^{\prime}\right) \in X_{b} ;
\end{aligned}
$$

hence, since also $(x, y) \in X_{b}$, we conclude that $\left(x^{\prime}, y^{\prime}\right) \in X_{a}$ as claimed.

6.4 Lawvere-complete $=$ Cauchy-complete. It is now straightforward to prove that the two notions of completeness coincide.

Theorem. For a quasi-uniform space $(X, A)$ the following conditions are equivalent.

(i) $(X, A)$ is a Lawvere-complete lax algebra.

(ii) $(X, A)$ is a Cauchy-complete quasi-uniform space.

Proof. (i) $\Rightarrow$ (ii): Each minimal Cauchy filter in $(X, A)$ defines an adjoint pair of bimodules $(\Phi: 1 \multimap(X, A)) \dashv(\Psi:(X, A) \multimap 1)$, which, by (i), is induced by a map $f: 1 \rightarrow X, \star \mapsto x_{0}$. Hence $\Phi=\left\{\varphi_{b}=b \cdot f \mid b \in B\right\}$ and $\Psi=\left\{\psi_{b}=f^{\circ} \cdot b \mid b \in B\right\}$. Moreover, $x \in X_{\star \varphi_{b}-}$ exactly when $b\left(x_{0}, x\right)=\top$, that is $X_{\star \varphi_{b}-}=X_{x_{0} b-}$, and $x \in X_{-\psi_{b} \star}$ exactly when $b\left(x, x_{0}\right)=\top$, which means $X_{-\psi_{b} \star}=X_{-b x_{0}}$.

(ii) $\Rightarrow$ (i): Given an adjoint pair of bimodules $(\Phi: 1 \multimap(X, A)) \dashv(\Psi:(X, A) \multimap 1)$, by (ii) the minimal Cauchy filter it induces is the neighbourhood filter of a point $x_{0}$. It is straightforward to check that $\Phi=A \cdot f$ and $\Psi=f^{\circ} \cdot A$ for $f: 1 \rightarrow X, \star \mapsto x_{0}$.

Final remark. The results of this section can be investigated in the more general setting introduced in [9], i.e., in proalgebras; here, for simplicity, we decided to state them only at the level of quasi-uniform structures, which are proalgebras for the identity monad. 


\section{References}

[1] M. Barr, Relational algebras, in: Springer Lecture Notes in Math. 137 (1970), pp. 39-55.

[2] J. Bénabou, Distributors at work, lecture notes written by Thomas Streicher, www.mathematik.tudarmstadt.de/ streicher/

[3] R. Betti, A. Carboni, R. Street and R. Walters, Variation through enrichment, J. Pure Appl. Algebra 29 (1983) 109-127.

[4] M.M. Bonsangue, F. van Breugel and J.J.M.M. Rutten, Generalized metric spaces: Completion, topology, and powerdomains via the Yoneda embedding, Theoret. Comput. Sci. 193 (1998) 1-51.

[5] F. Borceux, Handbook of Categorical Algebra, Vol. 1, Cambridge Univ. Press 1994.

[6] F. Borceux and D. Dejean, Cauchy completion in category theory, Cahiers Topologie Géom. Différentielle Catég. 27 (1986) 133-146.

[7] M.M. Clementino and D. Hofmann, Topological features of lax algebras, Appl. Categ. Structures 11 (2003) 267-286.

[8] M.M. Clementino and D. Hofmann, On extensions of lax monads, Theory Appl. Categ. 13 (2004) 41-60.

[9] M.M. Clementino, D. Hofmann and W. Tholen, One setting for all: metric, topology, uniformity, approach structure, Appl. Categ. Structures 12 (2004) 127-154.

[10] M.M. Clementino and W. Tholen, Metric, Topology and Multicategory - A common approach, J. Pure Appl. Algebra 179 (2003) 13-47.

[11] S. Eilenberg and G.M. Kelly, Closed categories, In Proc. Conf. Categorical Algebra (La Jolla, Calif., 1965), 421-562, Springer, New York, 1966.

[12] P. Fletcher and W. Lindgren, A construction of the pair-completionf a quasi-uniform space, Canad. Math. Bull. 21 (1978), 53-59.

[13] P. Fletcher and W. Lindgren, Quasi-uniform spaces, Lecture Notes in Pure and Applied Maths, Marcel Dekker ed, 1982.

[14] P. Freyd and A. Scedrov, Categories, allegories, volume 39 of North-Holland Mathematical Library, North-Holland Publishing Co., Amsterdam, 1990.

[15] D. Hofmann, An algebraic description of regular epimorphisms in topology, J. Pure Appl. Algebra 199 (2005) 71-86.

[16] D. Hofmann, Topological theories and closed objects, Preprint CM06 I-35 (2006), University of Aveiro.

[17] G.M. Kelly, Basic concepts of enriched category theory, volume 64 of London Mathematical Society Lecture Note Series, Cambridge University Press, Cambridge, 1982.

[18] F.W. Lawvere, Metric spaces, generalized logic, and closed categories, Rend. Sem. Mat. Fis. Milano 43 (1973) 135-166; Reprints in Theory and Applications of Categories 1 (2002) 1-37.

[19] R. Lowen, Approach spaces, Oxford Mathematical Monographs, The Clarendon Press Oxford University Press, New York, 1997.

[20] M. Mahmoudi, C. Schubert and W. Tholen, Universality of coproducts in categories of lax algebras, Appl. Categ. Structures 14 (2006) 243-249.

[21] E. Manes, Taut monads and T0-spaces, Theoret. Comput. Sci. 275 (2002) 79-109. 
[22] I. Moerdijk, Monads on tensor categories. J. Pure Appl. Algebra 168, 2-3 (2002), 189-208.

[23] C. Van Olmen, A study of the interaction between frame theory and approach theory, $\mathrm{PhD}$ thesis, University of Antwerp, 2005.

[24] G.J. Seal, Canonical and op-canonical lax algebras, Theory Appl. Categ. 14 (2005) 221-243 (electronic).

[25] W. Tholen, Lectures on Lax-Algebraic Methods in General Topology, Lecture 1: V-categories, V-modules, Lawvere completeness, Haute-Bodeux (2007), http://www.math.yorku.ca/ tholen/HB07Tholen1.pdf

[26] R.J. Wood, Abstract proarrows. I. Cahiers Topologie Géom. Différentielle 23 (1982), no. 3, 279-290.

[27] R.J. Wood, Ordered sets via adjunctions, in: Categorical foundations, volume 97 of Encyclopedia Math. Appl., pages 5-47. Cambridge Univ. Press, Cambridge, 2004.

Maria Manuel Clementino

CMUC/ Department of Mathematics

University of Coimbra

3001-454 Coimbra, PORTUGAL

mmc@mat.uc.pt
Dirk Hofmann

UIMA/Department of Mathematics

University of Aveiro

3810-193 Aveiro, PORTUGAL

dirk@mat.ua.pt 